\title{
Simple Connectivity in Polar Spaces with Group-Theoretic Applications
}

\author{
Reed Nessler \\ Charlottesville, Virginia
}

B.A., University of Virginia, 2007

\begin{abstract}
A Dissertation presented to the Graduate Faculty of the University of Virginia in Candidacy for the Degree of Doctor of Philosophy
\end{abstract}

Department of Mathematics

University of Virginia

August 2014 


\title{
Simple Connectivity in Polar Spaces with Group-Theoretic Applications
}

by

Reed Nessler

Submitted to the Department of Mathematics on August 1, 2014, in partial fulfillment of the requirements for the Degree of

Doctor of Philosophy

\begin{abstract}
In this dissertation, I prove that $\Delta^{0}(C)$ is simply connected, for any chamber $C$ of a nonembeddable polar space $\Delta$, using a recent coordinate description of these spaces. This fact is used in describing some group amalgamations in the setting of BN-pairs and related structures. Finally I discuss some progress in studying $\Delta^{0}(C)$ in a building of type $\mathrm{F}_{4}$.
\end{abstract}

Supervisor: Peter Abramenko

Title: Professor 


\section{Acknowledgments}

First of all I sincerely thank my advisor Prof. Peter Abramenko for his continuous support and assistance, and for finding me an interesting mathematical problem to solve.

I also want to thank the rest of my dissertation committee-Prof. Andrei Rapinchuk, Prof. John Faulkner, and Prof. Despina Louca-for their participation.

Acknowledgment is due to Prof. Hendrik Van Maldeghem for improvements to the exposition, and of course for co-creating the coordinate system that enabled this work.

I dedicate this dissertation to the late Prof. Bob Stong, without whose support at a crucial time it never would have been written. 


\section{Contents}

0.1 Introduction . . . . . . . . . . . . . . . . 10

1 Background 13

1.1 Simplicial complexes and chamber complexes . . . . . . . . . 13

1.2 Coxeter complexes . . . . . . . . . . . . . . . . 14

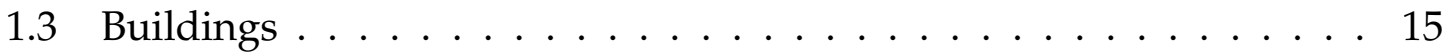

1.4 The $\delta$ function and twin buildings . . . . . . . . . . . . 17

1.5 Buildings and groups . . . . . . . . . . . . . . . . . 19

1.6 Embeddable and nonembeddable polar spaces . . . . . . . . . . 20

2 Simple connectivity in polar spaces 23

2.1 Point-line geometries . . . . . . . . . . . . . 23

2.2 Nonembeddable polar spaces . . . . . . . . . . . . . . 24

2.3 Proof of the main theorem . . . . . . . . . . . . 28

3 Group-theoretic applications $\quad 41$

3.1 First results . . . . . . . . . . . . . . . . . 41

3.2 An application involving twin buildings . . . . . . . . . . . 42

3.3 Finite presentability of $B_{ \pm} \ldots \ldots \ldots \ldots$. . . . . . . . . 47

4 Some remarks on type $\mathrm{F}_{4} \quad 49$

4.1 The $\mathrm{E}_{6}$ and $\mathrm{F}_{4}$ geometries $\ldots \ldots \ldots \ldots . \ldots \ldots$

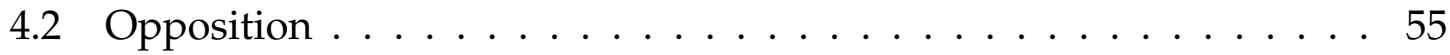

4.3 Viewing $\mathcal{M}^{\rangle\langle q\rangle}$ as a classical polar space . . . . . . . . . . 58 
4.4 More results $\ldots \ldots \ldots \ldots \ldots \ldots$ 


\section{List of Figures}

4-1 The solid lines form the collinearity graph of a point $\langle q\rangle$ (central dot) and its eight neighboring points in an apartment. Also shown, dotted, are three of the six hyperlines containing $\langle q\rangle$ in the apartment. 61 


\subsection{Introduction}

A finite reflection group naturally acts on the unit sphere, which becomes a simplicial complex when triangulated by the hyperplanes corresponding to the reflections. The distance between two maximal simplices, or chambers, is measured by counting the number of hyperplanes separating them; this definition coincides with their so-called gallery distance as defined in section 1.1. Thus the set of chambers is a metric space. It has finite diameter: when two chambers are antipodal on the sphere, their distance reaches a maximum value, which is the length of the longest element $w_{0}$ of the reflection group. Pairs of chambers at this distance are called opposite.

A spherical building $\Delta$ is a union of such spheres, its apartments. The distance between two chambers in $\Delta$ is measured in any apartment containing both. In this way the chamber set of $\Delta$ becomes a metric space with the same diameter as its apartments, and again chambers are opposite if they attain the diameter. For any chamber $C \in \Delta$, we define $\Delta^{0}(C)$ as the complex generated by the chambers opposite $C$.

$\Delta$ itself has the homotopy type of a wedge of spheres, according to the SolomonTits theorem. That is, if $\operatorname{dim} \Delta=n$ then $\Delta$ is $n$-spherical, meaning $(n-1)$-connected. As a "large" subcomplex of $\Delta, \Delta^{0}(C)$ too is expected to be $n$-spherical, but there are exceptions.

Peter Abramenko was originally motivated to study $\Delta^{0}(C)$ by the occurrence of spherical buildings as links in Bruhat-Tits buildings associated to $S$-arithmetic groups. Topological properties of $\Delta^{0}(C)$ lead to topological properties of invariant subcomplexes of the Bruhat-Tits building, and thence to homological finiteness properties of the group [2].

A more elementary motivation to study $\Delta^{0}(C)$ involves groups with BN-pair of spherical type, i.e. finite Weyl group. Such a group always acts on an associated spherical building, and the action restricts to an action of $B$ on $\Delta^{0}(C)$ with a chamber as fundamental domain. Provided that $\Delta^{0}(C)$ is simply connected, a theorem of 
Soulé [12] then expresses $B$ as an amalgam of its subgroups $B \cap P$, where $P$ runs through the maximal subgroups of $G$ containing $w_{0} B w_{0}$.

More generally, simple connectedness of $\Delta^{0}(C)$ leads to amalgam presentations of the subgroups $B_{ \pm}$in a group $G$ with twin BN-pair, where this time $\Delta$ is the associated twin building, which is a pair of buildings having an opposition relation between them. In this dissertation we show that $B_{ \pm}$are finitely presented if $G=$ $G\left(\mathbb{F}_{q}\right)$ is a Kac-Moody group with $5 \leq q<\infty$, under suitable conditions on $G$.

In [2], $\Delta^{0}(C)$ was shown to be $(n-1)$-spherical for all "sufficiently thick" buildings of types $A_{n}, C_{n}$, and $D_{n}$, excluding the exceptional $C_{3}$ buildings arising from so-called nonembeddable polar spaces. These spaces, first constructed by Tits based on the classification of algebraic simple groups [13], lacked an explicit description suitable for computations, and thus this case remained open ever since. In this dissertation, we prove as our main theorem that $\Delta^{0}(C)$ is 2-spherical (= simply connected), for any chamber $C$ of an exceptional $C_{3}$ building $\Delta$, using the recent coordinate description by De Bruyn and Van Maldeghem [7].

This dissertation is structured as follows. The first chapter collects necessary background material on buildings. In chapter 2 we describe the coordinates for the nonembeddable polar spaces and prove the main theorem. Chapter 3 presents some group-theoretic applications of the theorem. The final chapter reports some preliminary work in studying $\Delta^{0}(C)$ in the case of the $\mathrm{F}_{4}$ building associated to a field, itself depending on an $\mathrm{E}_{6}$ construction. 


\section{Chapter 1}

\section{Background}

The main reference for the material presented in this chapter is [3].

\subsection{Simplicial complexes and chamber complexes}

By an abstract simplicial complex $\Delta$ with vertex set $\mathcal{V}$ we understand a nonempty collection of finite subsets of $\mathcal{V}$ (the simplices), which must include all singletons and all subsets of each simplex. The dimension of a simplex is one less than its cardinality as a set of vertices. There is a standard way of constructing a topological space $|\Delta|$ based on $\Delta$, called its geometric realization; when we speak of topological properties of $\Delta$, for instance $n$-sphericity, we have in mind properties of $|\Delta|$.

Recall that a binary relation $\leq$ is a partial order if for all elements $a, b$, and $c$ it satisfies $a \leq a$; if $a \leq b$ and $b \leq a$ then $a=b$; and if $a \leq b$ and $b \leq c$ then $a \leq c$. We call a set together with a partial order a poset. The relation of set containment provides a partial order on a simplicial complex $\Delta$, which we may write $\leq$ and interpret as "is a face of." With respect to $\leq$, any two simplices have a greatest lower bound (their intersection), and the set of faces of any simplex has the form of a finite power set. On the other hand, these properties in an arbitrary poset suffice to define a simplicial complex, as Tits does in [13]. A simplicial complex in the ordinary sense is recovered by defining a vertex as a poset element with exactly one minimal nonzero face (namely, itself), and then associating to each poset element 
$A$ the simplex $\{v \in \mathcal{V}: v \leq A\}$.

Any poset has a flag complex, which is the set of finite totally ordered subsets, partially ordered by inclusion. It is a simplicial complex in the sense just defined.

Suppose that all maximal simplices in a simplicial complex $\Delta$ have the same dimension, which we call the dimension of $\Delta$. Then a gallery in $\Delta$ is a sequence of maximal simplices with consecutive ones having 1-codimensional intersection. If every pair of maximal simplices can be connected by a gallery then $\Delta$ is a chamber complex. In that case we call the maximal simplices chambers and their 1-codimensional faces panels. The gallery distance between two chambers is one less than the minimum number of chambers among galleries connecting them. If every panel is the face of exactly two chambers then we call $\Delta$ thin.

A type function on a chamber complex is a function from $\mathcal{V}$ into a $(\operatorname{dim} \Delta+1)$ element set which is bijective on every chamber.

\subsection{Coxeter complexes}

The pair $(W, S)$ is a Coxeter system if $W$ is a group with presentation

$$
W=\left\langle S \mid\left(s_{i} s_{j}\right)^{m_{i j}}=1\right\rangle,
$$

where $m_{i i}=1$ and $m_{i j} \geq 2$ for $i \neq j . m_{i j}=\infty$ is allowed, and means "no relation." The rank of the Coxeter system is $|S|$.

If $(W, S)$ is a Coxeter system then the Coxeter complex $\Sigma=\Sigma(W, S)$ associated to $(W, S)$ is the set $\{w\langle J\rangle: w \in W, J \subseteq S\}$, partially ordered by reverse inclusion. So $w_{1}\langle J\rangle \leq w_{2}\langle K\rangle$ means $w_{1}\langle J\rangle \supseteq w_{2}\langle K\rangle$; we say that $w_{1}\langle J\rangle$ is a face of $w_{2}\langle K\rangle$. This poset is an abstract simplicial complex as defined in section 1.1; moreover, it is a thin chamber complex [3, Theorem 3.5].

If $W$ is finite, it is a finite reflection group and $\Sigma$ triangulates a sphere, which coincides with the complex mentioned in the introduction. In general, since the chambers are the cosets with $J=\varnothing$, there is a one-to-one correspondence $C(\Sigma) \leftrightarrow$ 
$W$, where $C(\Sigma)$ denotes the set of chambers of $\Sigma$. Panels are of the form $w\langle s\rangle$, which is the face of precisely the chambers $w$ and $w s$.

Additionally $\Sigma$ possesses the type function $\tau(w\langle J\rangle):=S \backslash J[3$, Theorem 3.5] and a Weyl distance function $\delta: C(\Sigma) \times C(\Sigma) \rightarrow W$ given by $\delta\left(w_{1}, w_{2}\right)=w_{1}^{-1} w_{2}$. It is the case that $d\left(w_{1}, w_{2}\right)=\ell\left(\delta\left(w_{1}, w_{2}\right)\right)$, where $\ell$ is the word metric with respect to $S[3$, Proposition 3.87].

$W$ is finite if and only if $\Sigma$ has finite diameter, or supremum of $d$. In this case, the diameter of $\Sigma$ is $\ell\left(w_{0}\right)$, and chambers at this gallery distance are called opposite. Here $w_{0}$ denotes the unique longest element with respect to $\ell$, which always satisfies $w_{0}^{2}=1$ and $w_{0} S w_{0}^{-1}=S[3$, Proposition 1.77(4)]. Simplices are opposite if they are contained in opposite chambers and have $w_{0}$-conjugate types.

\subsection{Buildings}

Definition (4.1 in [3]). A building is a simplicial complex $\Delta$ that can be expressed as the union of subcomplexes $\Sigma$ (called apartments) satisfying the following axioms:

1. Each apartment is a Coxeter complex.

2. For any two simplices $A, B \in \Delta$, there is an apartment containing both of them.

3. If $\Sigma$ and $\Sigma^{\prime}$ are two apartments containing $A$ and $B$, then there is an isomorphism $\Sigma \rightarrow \Sigma^{\prime}$ fixing $A$ and $B$ pointwise.

There is a fixed Coxeter complex $\Sigma(W, S)$ such that $\Sigma \cong \Sigma(W, S)$ for every apartment $\Sigma$ [3, Corollary 4.8]. The notions of panel, chamber, gallery distance, and (if $W$ is finite) opposition extend from the apartments to the building in a welldefined way, by choosing an apartment $\Sigma \cong \Sigma(W, S)$ containing a given simplex or pair of simplices. If an apartment $\Sigma_{0}$ and isomorphism $\Sigma_{0} \cong \Sigma(W, S)$ are fixed, then the type function that $\Sigma_{0}$ inherits from $\Sigma(W, S)$ extends uniquely to a type function on $\Delta[3$, Proposition 4.6]. 
A building is thick if every panel is contained in at least 3 chambers. It is spherical if $W$ is finite.

Buildings originated as a means of giving a geometric interpretation to the semisimple Lie groups. "Geometric" in this context refers to incidence geometry, and indeed buildings serve to unify and generalize several classes of geometry, including projective spaces (type $A_{n}$ ) and polar spaces (type $C_{n}$ ). More precisely, an incidence geometry is a poset satisfying particular axioms, and the building arises as the flag complex of this poset.

We will consider polar spaces in section 1.6. Projective spaces, though they may be axiomatized directly as posets (in lattice-theoretic jargon, "projective space" means "simple complemented modular lattice" [4, §70]), are perhaps more commonly encountered in the guise of point-line geometry. (For some relevant definitions, see section 2.1.) We begin with the familiar axioms for a projective plane:

1. Any two points are incident to a unique line.

2. Any two lines are incident to a unique point.

3. There exist three noncollinear points.

A projective space, then, is defined by the following axioms [5, Definition 5.2.1]:

1. Any two points are incident to a unique line.

2. The subspace generated by any three noncollinear points is a projective plane.

3. There exist three noncollinear points.

We regard a projective space as a poset by forming the set of all points, lines, and any higher-dimensional proper subspaces, ordered by inclusion. The flag complex of an $n$-dimensional projective space is a building of type $\mathrm{A}_{n}$, and conversely, every building of type $\mathrm{A}_{n}$ is isomorphic to the flag complex of an $n$-dimensional projective space, unique up to isomorphism [13, Theorem 6.3].

Most familiar are the Desarguesian projective spaces: if $V$ is a vector space of finite dimension $n \geq 2$ over a skew field, then $\mathbf{P}(V)$ comprises the set of proper 
nonzero subspaces of $V$, ordered by inclusion. Its flag complex is a building of type $\mathrm{A}_{n-1}$. Every thick projective space of dimension greater than two is Desarguesian, but there are non-Desarguesian projective planes.

\subsection{The $\delta$ function and twin buildings}

In a building $\Delta$ of type $(W, S)$ there is a Weyl distance function $\delta: C(\Delta) \times C(\Delta) \rightarrow W$ between pairs of chambers. It can be defined by choosing an apartment $\Sigma \cong \Sigma(W, S)$

containing two chambers and using the $\delta$ function as previously defined for $\Sigma(W, S)$ [3, Definition 4.82]. This definition does not depend on the choice of apartment or isomorphism [3, Proposition 4.81].

It can be shown that $\delta$ satisfies the following properties [3, Proposition 4.84]. Here $C$ and $D$ are arbitrary chambers of $\Delta$.

1. $\delta(C, D)=1$ if and only if $C=D$.

2. If $\delta(C, D)=w$ and $C^{\prime} \in C(\Delta)$ satisfies $\delta\left(C^{\prime}, C\right)=s \in S$, then $\delta\left(C^{\prime}, D\right) \in\{s w, w\}$. If also $\ell(s w)=\ell(w)+1$ then $\delta\left(C^{\prime}, D\right)=s w$.

3. If $\delta(C, D)=w$, then for any $s \in S$ there is a chamber $C^{\prime} \in C(\Delta)$ such that $\delta\left(C^{\prime}, C\right)=s$ and $\delta\left(C^{\prime}, D\right)=s w$.

On the other hand, given a set $C$ and a function $\delta: C \times C \rightarrow W$ satisfying these properties, there exists a building, unique up to isomorphism, such that $C=C(\Delta)$ with $\delta$ as its Weyl distance function [3, Corollary 5.93]. The simplices in this building correspond to the residues of $C$, which are certain distinguished sets of chambers; for a precise description see $[3, \S 5.6]$. Thus it is possible to define a building as a pair $(C, \delta)$ satisfying 1.-3. as axioms.

We adopt this point of view in defining twin buildings, which historically arose from the study of Kac-Moody groups much as algebraic groups lead to buildings.

Definition (5.133 in [3]). A twin building of type $(W, S)$ is a triple $\left(C_{+}, C_{-}, \delta^{*}\right)$ consisting of two buildings $\left(C_{+}, \delta_{+}\right)$and $\left(C_{-}, \delta_{-}\right)$of type $(W, S)$ together with a 
codistance function

$$
\delta^{*}:\left(C_{+} \times C_{-}\right) \cup\left(C_{-} \times C_{+}\right) \rightarrow W
$$

satisfying the following conditions for each $\epsilon \in\{+,-\}$, any $C \in C_{\epsilon}$, and any $D \in C_{-\epsilon}$, where $w:=\delta^{*}(C, D)$ :

1. $\delta^{*}(C, D)=\delta^{*}(D, C)^{-1}$.

2. If $C^{\prime} \in C_{\epsilon}$ satisfies $\delta_{\epsilon}\left(C^{\prime}, C\right)=s$ with $s \in S$ and $\ell(s w)<\ell(w)$, then $\delta^{*}\left(C^{\prime}, D\right)=$ sw.

3. For any $s \in S$, there exists a chamber $C^{\prime} \in C_{\epsilon}$ with $\delta_{\epsilon}\left(C^{\prime}, C\right)=s$ and $\delta^{*}\left(C^{\prime}, D\right)=$ $s w$.

Notwithstanding this choice of definition, we will find it useful to think of the buildings $\left(C_{ \pm}, \delta_{ \pm}\right)$as simplicial complexes $\Delta_{ \pm}$.

Twin buildings generalize spherical buildings. Given a spherical building $(C, \delta)$, one obtains a twin building by defining $C_{ \pm}$to be disjoint copies of $C, \delta_{+}:=\delta$, $\delta_{-}:=w_{0} \delta w_{0}, \delta^{*}\left(C_{+}, D_{-}\right):=\delta(C, D) w_{0}$, and $\delta^{*}\left(D_{-}, C_{+}\right):=w_{0} \delta(D, C)$ [3, Example 5.136(a)]. And conversely, every twin building with $W$ finite is of this form [3, Exercise 5.163].

Like spherical buildings, twin buildings have a notion of opposition. Chambers $C_{ \pm} \in C_{ \pm}$are opposite when they lie at codistance $1 \in W$, and simplices of a twin building are opposite if they are contained in opposite chambers and have the same type. Opposite chambers are contained in a unique twin apartment, a pair of apartments on which opposition is bijective [3, Proposition 5.179(1)].

In the twin building associated to a spherical building, the fact that $\delta_{-}$and $\delta_{+}$ are $w_{0}$-conjugate, when interpreted from the simplicial point of view, implies that "same type" for simplices in different halves of the twin building coincides with " $w_{0}$-conjugate type" in the spherical building. In particular, the two definitions of "opposite" agree with each other.

Definition. Suppose $\Delta$ is a spherical building or twin building and $C$ is a chamber of $\Delta$. The complex $\Delta^{0}(C)$ consists of all chambers opposite $C$ and their faces. 


\subsection{Buildings and groups}

We assume that a group acting on a building acts by simplicial automorphisms that preserve type; an action on a twin building is a pair of actions on $\Delta_{ \pm}$preserving codistance. An action on a building is strongly transitive with respect to a system of apartments if transitive on the set of pairs $(\Sigma, C)$, where $\Sigma$ is an apartment and $C$ is a chamber of $\Sigma$ [3, Definition 6.1]. If the building is spherical, this is equivalent to being transitive on pairs of opposite chambers [3, Proposition 6.15], which we may take as the definition of strongly transitive when describing a group action on a twin building.

The study of groups acting on buildings has led to characterizations of these actions internally, in terms of subgroups subject to axioms:

- A strongly transitive action on a thick building corresponds to a BN-pair; the building is recovered from the BN-pair as the set $\Delta(G, B)$ of standard parabolic cosets $g B W_{J} B(g \in G, J \subseteq S)$, ordered by reverse inclusion.

- A strongly transitive action on a thick spherical Moufang building corresponds to an RGD system of spherical type.

- A strongly transitive action on a thick twin building corresponds to a twin BN-pair; its two halves are buildings $\Delta\left(G, B_{ \pm}\right)$as constructed above.

- A strongly transitive action on a thick Moufang twin building corresponds to an RGD system (not necessarily spherical).

Let us define some of these terms.

Definition (6.55 in [3]). Subgroups $B$ and $N$ of a group $G$ form a BN-pair if they generate $G$, the intersection $T:=B \cap N$ is normal in $N$, and the quotient $W:=N / T$ (the Weyl group) admits a set of generators $S$ such that the following hold:

1. For $s \in S$ and $w \in W, s B w \subseteq B s w B \cup B w B$.

2. For $s \in S, s B s^{-1} \not \leq B$. 
In this case $(G, B, N, S)$ is called a Tits system.

A standard example is $G=\mathrm{GL}_{n}(k)$, with $B$ the upper-triangular group and $N$ the monomial group, those matrices with exactly one nonzero entry in every row and column. Here $W$ is isomorphic to the group of permutation matrices, and $S$ can comprise the set of adjacent transpositions.

Definition (6.78 in [3]). Let $B_{+}, B_{-}$, and $N$ be subgroups of a group $G$ such that $B_{+} \cap N=B_{-} \cap N=: T$. Assume that $T \unlhd N$, and set $W:=N / T$. The triple $\left(B_{+}, B_{-}, N\right)$ is called a twin BN-pair with Weyl group $W$ if $W$ admits a set $S$ if generators such that the following conditions hold for all $w \in W$ and $s \in S$ and each $\epsilon \in\{+,-\}$ :

1. $\left(G, B_{\epsilon}, N, S\right)$ is a Tits system.

2. If $\ell(s w)<\ell(w)$, then $B_{\epsilon} s B_{\epsilon} w B_{-\epsilon}=B_{\epsilon} s w B_{-\epsilon}$.

3. $B_{+} S \cap B_{-}=\varnothing$.

The definition of Moufang is technical and will not be needed below; it generalizes the notion of Moufang plane from projective geometry. For definitions of RGD systems, see [3, §7.8 and §8.6].

\subsection{Embeddable and nonembeddable polar spaces}

Like projective spaces, polar spaces may be defined by giving axioms either to points and lines (as we do in section 2.1) or to the poset of subspaces [13, §7.1]. The most familiar example of a polar space arises as follows. Suppose $V$ is a vector space over a field of characteristic not 2 , equipped with a nondegenerate symmetric bilinear form $(\cdot, \cdot)$. We define $M^{\perp}:=\{x \in V:(x, M)=0\}$ for any subset $M \subseteq V$ and call a subspace $U \leq V$ totally isotropic if $U \subseteq U^{\perp}$. Assume that the maximal dimension $n$ of a totally isotropic subspace is finite. Then $\{0<$ $U<V: U$ is totally isotropic\} is a polar space of rank $n$. By construction, it comes

embedded in a Desarguesian projective space. In general, a polar space is called embeddable if it can be embedded in a projective space, nonembeddable if not. 
The above example can be generalized to encompass vector spaces over skew fields of arbitrary characteristic, by introducing hermitian and pseudoquadratic forms. Every thick embeddable polar space of rank at least 3 can be constructed in this way [13, Theorem 8.22]. The sphericity proof for $\Delta^{0}(C)$ in case $C_{n}$ in [2] relies on this explicit description of the embeddable polar spaces.

Most polar spaces are embeddable. Among the thick polar spaces of rank at least 3, every nonembeddable polar space has rank 3 and arises from a single construction involving some Cayley-Dickson division algebra. We now turn to a more detailed look at these nonembeddable polar spaces. 


\section{Chapter 2}

\section{Simple connectivity in polar spaces}

\subsection{Point-line geometries}

The following definitions are taken from $[6, \S 1]$. A space is a pair $(P, L)$ where $P$ is a nonempty set whose elements are called points and $L$ is a collection of subsets of $P$ of cardinality at least 2 , called lines. Points $x, y \in P$ are collinear, written $x \perp y$, if there exists $l \in L$ with $x, y \in l$. A set $X \subseteq P$ is called a subspace of $(P, L)$ if every line $l \in L$ meeting $X$ in at least two points entirely belongs to $X$ and is called singular if any two of its points are collinear. We call a space nondegenerate if no point is collinear with all other points. The spaces we will consider have the property that any pair of distinct collinear points is contained in a unique line; these are the partial linear spaces. The singular rank of a space is the maximal number $n$ (possibly $\infty$ ) for which there exists a chain of distinct subspaces $\varnothing=X_{-1} \subsetneq X_{0} \subsetneq X_{1} \cdots \subsetneq X_{n}$ such that $X_{i}$ is a singular subspace for each $i(0 \leq i \leq n)$. For example, the singular rank of a projective space is its dimension.

A morphism $(P, L) \rightarrow\left(P^{\prime}, L^{\prime}\right)$ of spaces is a function $P \rightarrow P^{\prime}$ for which the image of every line in $L$ is contained in a line in $L^{\prime}$. Isomorphism is defined in the obvious way.

The collinearity graph of a space $(P, L)$ is the graph with vertex set $P$ and having an edge joining each pair of distinct collinear points.

A proper subspace $X \subseteq P$ is a hyperplane if every line of $X$ has at least one point 
in X. A (nondegenerate) polar space may then be defined as a space in which for every point $x$, the set $x^{\perp}$ of points collinear with $x$ is a hyperplane. It is a fact that every singular subspace of a polar space is a projective space [6, Theorem 3.1]; if the maximal dimension of a singular subspace is $n-1$ (i.e. the singular rank is $n-1$ ) then the polar space has rank $n$.

The collection of singular subspaces of a rank- $n$ polar space, with two of them declared incident if one contains the other, gives rise to a flag complex $\Delta$ that is a building of type $C_{n}$; conversely, every building of type $C_{n}$ arises in this way [13, $\S 7.4]$.

We can describe the opposition relation in $\Delta$ in terms of the underlying polar space. Vertices $A, B \in \Delta$ are opposite if, as subspaces, no point of one is collinear with all points of the other. Two flags are opposite if every member of one flag is opposite some member of the other flag. Let $C$ be a chamber of $\Delta$. Then $\Delta^{0}(C)$ is the subcomplex of $\Delta$ generated by the chambers opposite $C$. More generally, if $F$ is a flag of $\Delta$, then $\Delta^{0}(F)$ is generated by the chambers containing a flag opposite $F$.

\subsection{Nonembeddable polar spaces}

Let $\mathbb{K}$ be a commutative field, and let $\mathrm{O}$ be a Cayley-Dickson division algebra with center $\mathbb{K}$. The Moufang projective plane over $\mathrm{O}$ is the projective plane $\mathrm{PG}(2, \mathrm{O})$ with point set $\{(\infty),(m),(x, y): m, x, y \in \mathrm{O}\}$ and line set $\{[\infty],[x],[m, k]: x, m, k \in \mathrm{O}\}$, and with incidence relation $*$ defined by $(x, y) *[x] *(\infty) *[\infty] *(m) *[m, k]$ and

$$
(x, y) *[m, k] \text { if and only if } y=m x+k \text {. }
$$

According to Tits [13], there exists a unique polar space $\Delta$ of rank 3 with planes isomorphic to the Moufang projective plane over $\mathrm{O}$. As the planes are non-Desarguesian, such a polar space cannot be embedded in a projective space, i.e. is nonembeddable. Moreover, every thick nonembeddable polar space is of this form [6, Theorem 3.34]. We give the coordinate description which can be found in 
[7].

Let $\infty$ be a symbol not belonging to $O$. Then the point set of $\Delta$ is the set

$\left\{(\infty),\left(x_{1}\right),\left(x_{1}, x_{2}\right),\left(x_{1}, x_{2} ; k\right),\left(x_{1}, x_{2}, x_{3} ; k\right),\left(x_{1}, x_{2}, x_{3}, x_{4} ; k\right): x_{1}, x_{2}, x_{3}, x_{4} \in \mathrm{O}, k \in \mathbb{K}\right\}$.

The point $(\infty)$ is called the point of type 0 . If $x_{1}, x_{2}, x_{3}, x_{4} \in \mathbb{O}$ and $k \in \mathbb{K}$, then $\left(x_{1}\right)$ is called a point of type $1,\left(x_{1}, x_{2}\right)$ is called a point of type $2,\left(x_{1}, x_{2} ; k\right)$ is called a point of type $3,\left(x_{1}, x_{2}, x_{3} ; k\right)$ is called a point of type 4 and $\left(x_{1}, x_{2}, x_{3}, x_{4} ; k\right)$ is called a point of type 5 . The planes of $\Delta$ are the following subsets of points, subdivided into eight types, where in each case $a, b, s \in \mathbb{O}$. We use $\sigma$ to denote the standard involution of O.

Type I. The plane $[\infty]$ consists of the following points:

$$
\begin{aligned}
p_{[\infty], 1}(a, b) & :=(a, b), \\
p_{[\infty], 2}(s) & :=(s), \\
p_{[\infty], 3} & :=(\infty) .
\end{aligned}
$$

Type II. For every $k \in \mathbb{K}$, the plane $[k]$ consists of the following points:

$$
\begin{aligned}
p_{[k], 1}(a, b) & :=(a, b ; k), \\
p_{[k], 2}(s) & :=(s), \\
p_{[k], 3} & :=(\infty) .
\end{aligned}
$$

Type III. For every $x \in O$ and every $k \in \mathbb{K}$, the plane $[x ; k]$ consists of the following points:

$$
\begin{aligned}
p_{[x ; k], 1}(a, b) & :=(x, a, b ; k), \\
p_{[x ; k], 2}(s) & :=\left(-x^{\sigma}, s\right), \\
p_{[x ; k], 3} & :=(\infty) .
\end{aligned}
$$


Type IV. For every $x \in \mathrm{O}$ and all $k, l \in \mathbb{K}$, the plane $\alpha:=[x ; k, l]$ consists of the following points:

$$
\begin{aligned}
p_{\alpha, 1}(a, b) & :=\left(a, x+l a, b ; k+x^{\sigma} a+a^{\sigma} x+l a^{\sigma+1}\right), \\
p_{\alpha, 2}(s) & :=\left(x^{\sigma}, s ; l\right) \\
p_{\alpha, 3} & :=(\infty) .
\end{aligned}
$$

Type V. For all $x_{1}, x_{2} \in \mathbb{O}$ and every $k \in \mathbb{K}$, the plane $\alpha:=\left[x_{1}, x_{2} ; k\right]$ consists of the following points:

$$
\begin{aligned}
p_{\alpha, 1}(a, b) & :=\left(-x_{2}^{\sigma},-x_{1}^{\sigma}, a, b ; k\right), \\
p_{\alpha, 2}(s) & :=\left(s, x_{1}+x_{2} s\right), \\
p_{\alpha, 3} & :=\left(x_{2}\right) .
\end{aligned}
$$

Type VI. For all $x_{1}, x_{2} \in \mathrm{O}$ and all $k, l \in \mathbb{K}$, the plane $\alpha:=\left[x_{1}, x_{2} ; k, l\right]$ consists of the following points:

$$
\begin{aligned}
p_{\alpha, 1}(a, b) & :=\left(-x_{2}^{\sigma}, a, x_{1}^{\sigma}+k a, b ; l+x_{1} a+a^{\sigma} x_{1}^{\sigma}+k a^{\sigma+1}\right), \\
p_{\alpha, 2}(s) & :=\left(s, x_{1}+x_{2} s ; k\right), \\
p_{\alpha, 3} & :=\left(x_{2}\right) .
\end{aligned}
$$

Type VII. For all $x_{1}, x_{2}, x_{3} \in O$ and all $k, l \in \mathbb{K}$, the plane $\alpha:=\left[x_{1}, x_{2}, x_{3} ; k, l\right]$ consists of the following points:

$$
\begin{aligned}
p_{\alpha, 1}(a, b) & :=\left(a,-x_{3}^{\sigma}+x_{1} a, b, x_{2}^{\sigma}+k a-x_{1}^{\sigma} b ; l+x_{2} a+a^{\sigma} x_{2}^{\sigma}+k a^{\sigma+1}\right), \\
p_{\alpha, 2}(s) & :=\left(x_{1}, s, x_{2}+x_{3} s ; k\right), \\
p_{\alpha, 3} & :=\left(-x_{1}^{\sigma}, x_{3}\right) .
\end{aligned}
$$


Type VIII. For all $x_{1}, x_{2}, x_{3} \in \mathbb{O}$ and all $k, l, m \in \mathbb{K}$, the plane $\alpha:=\left[x_{1}, x_{2}, x_{3} ; k, l, m\right]$ consists of the following points:

$$
\begin{aligned}
p_{\alpha, 1}(a, b):= & \left(a, b, x_{3}{ }^{\sigma}+l b+x_{1} a, x_{2}{ }^{\sigma}+k a+x_{1}^{\sigma} b ;\right. \\
& \left.m+x_{2} a+a^{\sigma} x_{2}^{\sigma}+x_{3} b+b^{\sigma} x_{3}{ }^{\sigma}+k a^{\sigma+1}+l b^{\sigma+1}+\left(a^{\sigma} x_{1}^{\sigma}\right) b+b^{\sigma}\left(x_{1} a\right)\right), \\
p_{\alpha, 2}(s):= & \left(s, x_{1}+l s, x_{2}+x_{3} s ; k+x_{1}^{\sigma} s+s^{\sigma} x_{1}+l s^{\sigma+1}\right), \\
p_{\alpha, 3}:= & \left(x_{1}^{\sigma}, x_{3} ; l\right) .
\end{aligned}
$$

Each of the above point sets admits a natural bijection $\beta_{\alpha}$ onto the point set of the plane $\mathrm{PG}(2, \mathrm{O})$ by mapping the point $p_{\alpha, 1}(a, b)$ to the point $(a, b)$, the point $p_{\alpha, 2}(s)$ to the point $(s)$, and the point $p_{\alpha, 3}$ to $(\infty)$. Now, these planes determine the collinearity relation in $\Delta$, and collinearity determines the lines. It is proved in [7] that one can explicitly describe all lines of $\Delta$ as the inverse images of the point sets of the lines in $\mathrm{PG}(2, \mathrm{O})$ under the bijections $\beta_{\alpha}$. Moreover, the collection of all planes above is precisely the family of all maximal singular subspaces of $\Delta$.

In particular, one can check that the intersection of two planes is either empty, a single point, or a line of $\Delta$. In [7], all lines are explicitly given independently of the planes as sets of points, and the lines are subdivided into twelve types. Here, we will only need two of those types. The lines of type $L$ are the lines of the planes $\alpha$ of type VIII not containing $p_{\alpha, 3}$, and the lines of type $K$ are the lines of the planes $\alpha$ of type VIII containing $p_{\alpha, 3}$ but not $p_{\alpha, 2}(0)$. Note that each line of type $\mathrm{L}$ also occurs in a (unique) plane of type VII, and each line of type K occurs in a (unique) plane of type VI. Two points will be called L-collinear if they are contained in a common line of type L; similarly for K-collinearity.

We define the chamber $C_{\infty}$ as the chamber consisting of the point $(\infty)$, the line through the points $(\infty)$ and $(0)$, and the unique plane $[\infty]$ of type I.

If two points $p$ and $q$ are collinear, then we write $p \perp q$, and if two singular subspaces $V$ and $W$ are contained in a common singular subspace, then we denote by $\langle V, W\rangle$ the unique singular subspace of smallest dimension containing both $V$ and $W$. 


\subsection{Proof of the main theorem}

Theorem 1. Let $\Delta$ be a thick polar space of rank 3 , and let $C$ be a chamber of $\Delta$. Then $\Delta^{0}(C)$ is simply connected if $\Delta$ is isomorphic to a nonembeddable polar space.

Let $\Delta$ be the nonembeddable thick polar space of rank 3 related to the CayleyDickson division algebra $\mathrm{O}$ with center the commutative field $\mathbb{K}$. We want to show that $\Delta^{0}\left(C_{\infty}\right)$ is simply connected. (Simple connectivity of $\Delta^{0}(C)$ for an arbitrary chamber $C$ will follow, since $\Delta$ has a chamber-transitive symmetry group.) We follow the general strategy of Abramenko [2], namely, we define a filtration of $\Delta^{0}\left(C_{\infty}\right)$ (i.e., a sequence of nested subgeometries whose union is $\Delta^{0}\left(C_{\infty}\right)$ ) that begins with a "large" contractible subgeometry and preserves simple connectivity at each step.

Note that it follows from the fact that the point $(\infty)$ belongs to all planes of type I, II, III and IV that a point is opposite ( $\infty)$ if and only if it has type 5. Likewise, it is easy to see that a plane is disjoint from the plane $[\infty]$ (and hence opposite) if and only if it has type VIII. Finally, it also follows that the lines opposite $\langle(\infty),(0)\rangle$ are precisely those of type L. Hence $\Delta^{0}\left(C_{\infty}\right)$ is the geometry induced by the set

$$
Y:=\{\text { points of type } 5\} \cup\{\text { lines of type } \mathrm{L}\} \cup\{\text { planes of type VIII }\}
$$

Before defining the filtration of $Y$ we record a result to be used later.

Proposition 1. Suppose that $\Gamma$ is a thick generalized quadrangle in which every point and every line is incident with at least $n^{2}+n+2$ elements, $n \in \mathbb{N}, n \geq 1$. If $Z$ is a nonempty set of points and lines of $\Gamma$ such that for any element $z$ of $Z$ at most $n$ elements incident with $z$ in $\Gamma$ do not belong to $Z$, then the geometry induced by $Z$ is connected.

Proof. Let $x, y$ be two elements of $Z$. Without loss of generality, we may assume $d(x, y)=3$. Indeed, if $d(x, y)=2$, then consider any element of $Z$ incident with $y$ not incident with $x$ (since there is a unique element in $\Gamma$ incident with both $x$ and $y$, and $n \geq 1$, such element certainly exists). If $d(x, y)=4$, then any element of $Z$ incident with $y$ is at distance 3 from $x$. 
Since $n^{2}+n+2-n \geq n+2$, we can select $n+1$ elements $x_{0}, x_{1}, \ldots, x_{n}$ of $Z$ incident with $x$ and at distance 4 from $y$. Since for each $x_{i}, i=0,1, \ldots, n$, there are at most $n$ elements incident with $x_{i}$ and not belonging to $Z$, and since for each such element there is a unique element at distance 2 and incident with $y$, there are at least $\left(n^{2}+n+1\right)-n(n+1) \geq 1$ elements $y_{0}$ incident with $y$ such that the unique elements $z_{i}$ at distance 2 from $y_{0}$ incident with $x_{i}, i=0,1, \ldots, n$, belong to $Z$. If $u_{i}$ denotes the unique element incident with both $z_{i}$ and $y_{0}, i=0,1, \ldots, n$, then, by assumption, at least one of these, say $u_{0}$, belongs to $Z$, and so the path $\left(x, x_{0}, z_{0}, u_{0}, y_{0}, y\right)$ belongs to $Z$.

For our purposes, we will mostly need the previous result in the following setting.

Corollary 1. Let $\Gamma$ be a generalized quadrangle such that every element is incident with infinitely many other elements. Let $n \geq 1$ be an arbitrary natural number, and let $a_{1}, a_{2}, \ldots, a_{n}$ be $n$ elements of $\Gamma$. Then $\bigcap_{i=1}^{n} \Gamma^{0}\left(a_{i}\right)$ is connected.

Proof. Assume that $x \in \bigcap_{i=1}^{n} \Gamma^{0}\left(a_{i}\right)$. Let $i \in\{1,2, \ldots, n\}$. If $x$ is opposite $a_{i}$, then $\Gamma_{x}$ is contained in $\Gamma^{0}\left(a_{i}\right)$. If $d\left(x, a_{i}\right)=3$, then the only element of $\Gamma_{x}$ not contained in $\Gamma^{0}\left(a_{i}\right)$ is the unique one at distance 2 from $a_{i}$. If $d\left(x, a_{i}\right) \leq 2$, then obviously $x \notin \Gamma^{0}\left(a_{i}\right)$, a contradiction. Hence at most $n$ elements incident with $x$ are not contained in $\bigcap_{i=1}^{n} \Gamma^{0}\left(a_{i}\right)$.

So the corollary will follow from Proposition 1 if we show that $\bigcap_{i=1}^{n} \Gamma^{0}\left(a_{i}\right)$ is nonempty. Let $\ell$ be maximal with the property that there exists $x \in \bigcap_{i=1}^{\ell} \Gamma^{0}\left(a_{i}\right)$. Then $\ell \geq 1$. Suppose for a contradiction that $\ell<n$. Then $d\left(x, a_{\ell+1}\right) \leq 2$. By the previous paragraph applied to $\bigcap_{i=1}^{\ell} \Gamma^{0}\left(a_{i}\right)$, we know that there exists $y \in$ $\bigcap_{i=1}^{\ell} \Gamma^{0}\left(a_{i}\right)$ with $y$ incident with $x$ and $d\left(y, a_{\ell+1}\right)=d\left(x, a_{\ell+1}\right)+1$. Hence, after at most three steps, we can find an element in $\bigcap_{i=1}^{\ell+1} \Gamma^{0}\left(a_{i}\right)$, contradicting the definition of $\ell$.

Proposition 2. Let $q$ be a point of type 5. Then it is contained in a unique plane $\alpha_{q}$ of type $V$ and in a unique line $L_{q}$ intersecting $\langle(\infty),(0)\rangle$ nontrivially. Moreover, in the generalized 
quadrangle $\Delta_{q}$, the chambers $C$ opposite the chamber $C_{q}:=\left\{L_{q}, \alpha_{q}\right\}$ are precisely those for which $C \cup\{q\}$ is opposite $C_{\infty}$, i.e., $\Delta_{q}^{0}\left(C_{q}\right)=\Delta^{0}\left(C_{\infty}\right) \cap \Delta_{q}$.

Proof. The first assertions follow from the fact that $q$ is opposite $(\infty)$. The last assertion follows directly from Theorem 3.28 and Proposition 3.29 of [13].

We will define the filtration of $Y$ step-by-step. We will have $Y_{0} \subseteq Y_{1} \subseteq \cdots \subseteq$ $Y_{9} \subseteq Y_{10}=Y$, and we start by defining $Y_{0}$.

Throughout, put $p:=(0,0,0,0 ; 0) \in Y$ and let $Y_{0} \subseteq Y$ comprise the following subspaces:

1. points of type $5 \mathrm{~L}$-collinear with $p$;

2. lines of type L containing a point of type $5 \mathrm{~L}$-collinear with $p$ and whose point of type 5 with first coordinate 0 is either

(a) not collinear with $p$, or

(b) K-collinear with $p$;

3. planes of type VIII containing a line as in 2(b) above.

The geometry $Y_{0}$ can be contracted onto $p$ since for any subspace $U$ in $Y_{0}$, the subspace defined by the points in $U$ collinear with $p$ also belongs to $Y_{0}$ (and we will denote that subspace by $U^{\perp p}$ ), and for any $U$ in $Y_{0}$ all of whose points are collinear with $p$, the subspace $\langle p, U\rangle$ also belongs to $Y_{0}$.

Concerning the points of $Y_{0}$ it is appropriate the note that every point of $Y_{0} \backslash\{p\}$ has nonzero first coordinate. Conversely, one easily checks that every point in $\Delta$ of type 5 with nonzero first coordinate and collinear with $p$, is L-collinear with $p$. This makes it rather easy to recognize points of $Y_{0}$.

The planes in $Y_{0}$ admit a simple characterization via coordinates.

Proposition 3. Let $\alpha=\left[x_{1}, x_{2}, x_{3} ; k, l, m\right]$. Then $\alpha \in Y_{0}$ if and only if either $(i) x_{2}=$ $x_{3}=m=0$ or (ii) $x_{3} \neq 0$ and $m \neq 0$. Moreover, $p \in \alpha$ in case (i) but not in case (ii). 
Proof. We have $p_{\alpha, 1}(0,0)=\left(0,0, x_{3}^{\sigma}, x_{2}^{\sigma} ; m\right)$, so $p \in \alpha$ (and hence $\left.\alpha \in Y_{0}\right)$ if and only if $x_{2}=x_{3}=m=0$. If $p \notin \alpha$, then $\alpha^{\perp p}$ is a line, and hence $\alpha \in Y_{0}$ if and only if $\alpha^{\perp p} \in Y_{0}$. But $\alpha^{\perp p}$ has type $\mathrm{L}$ if and only if $p_{\alpha, 3}$ is not collinear with $p$ (by definition of type L), and this happens if and only if $x_{3} \neq 0$ (indeed, if $x_{3}=0$, then the plane $\left[x_{1}, 0,0 ; 0, l, 0\right]$ contains both $p$ and $p_{\alpha, 3}$; if $x_{3} \neq 0$, then any plane $\beta$ of type VIII through $p_{\alpha, 3}$ has third coordinate nonzero, implying that the point $p_{\beta, 1}(0,0)$ has nonzero third coordinate). Noting that a point of type 5 with first coordinate 0 and collinear with $p$, is $K$-collinear with $p$ if and only if its second coordinate is distinct from 0 , we see that, if $x_{3} \neq 0$, the point of type 5 with first coordinate 0 in $\alpha^{\perp p}$ is K-collinear with $p$ if and only if $p_{\alpha, 1}(0,0)$ is not collinear with $p$, and this happens if and only if $m \neq 0$ (indeed, the points of type 5 with first and second coordinate 0 contained in a common plane of type V, VI or VII all have a common last coordinate, as is easily checked; if $m=0$, then $\left(0,0, x_{3}^{\sigma}, x_{2}^{\sigma} ; 0\right), x_{3} \neq 0$, and $(0,0,0,0 ; 0)$ are contained in the plane $[0,0 ; 0])$.

Now define $Y_{1}$ as follows (where $\mathbb{K}^{\times}=\mathbb{K} \backslash\{0\}$; we will also use $\mathbb{O}^{\times}=O \backslash\{0\}$ ):

$Y_{1}=Y_{0} \cup \bigcup_{m \in K^{x}}\{q \in Y: q$ is a point $\mathrm{K}$-collinear with and distinct from $(0,0,0,0 ; m)\}$.

The points $(0,0,0,0 ; m)$ have special properties.

Lemma 1. For all $m \in \mathbb{K}$ we have $(\infty)^{\perp} \cap(0,0,0,0 ; m)^{\perp}=(\infty)^{\perp} \cap p^{\perp}$. In particular, no point of type 5 collinear with $(0,0,0,0 ; 0)$ is collinear with $(0,0,0,0 ; m), m \in \mathbb{O}^{\times}$.

Proof. One easily calculates that

$$
(\infty)^{\perp} \cap(0,0,0,0 ; m)^{\perp}=\left\{(0),\left(x_{1}, 0\right),\left(x_{1}, 0 ; k\right),\left(x_{1}, x_{2}, 0 ; k\right): x_{1}, x_{2} \in \mathrm{O}, k \in \mathbb{K}\right\},
$$

which is independent of $m$.

Proposition 4. For any $q \in Y_{1} \backslash Y_{0}$, the subgeometry $Y_{0} \cap \Delta_{q}$ is connected.

Proof. Let $m \in \mathbb{K}^{\times}$. An arbitrary plane $\alpha$ of type VIII containing the point $p_{\alpha, 1}(0,0)=$ $(0,0,0,0 ; m)$ has coordinates $\left[x_{1}, 0,0 ; k, l, m\right], x_{1} \in \mathbf{O}, k, l \in \mathbb{K}$. An arbitrary point 
in that plane K-collinear with and distinct from $(0,0,0,0 ; m)$ is just an arbitrary point in $\alpha$ with first coordinate equal to 0 and second distinct from 0 , i.e., $q=$ $\left(0, b, l b, x_{1}^{\sigma} b ; m+l b^{\sigma+1}\right), b \neq 0$. Lemma 1 implies that $q \notin Y_{0}$.

By Lemma 1, a point of type unequal to 5 is collinear with $p$ if and only if it is collinear with $(0,0,0,0 ; m)$. Hence lines in $\Delta_{q} \cap Y$ opposite $\langle q,(0,0,0,0 ; m)\rangle$ are exactly those whose point of type 4 is not collinear with $p$. As $p$ is not collinear with $q$, such a line's unique point $r$ collinear with $p$ has type 5 . Moreover, the first coordinate of $r$ is nonzero as $r$ and $q$ are L-collinear but not K-collinear. It follows that $p$ and $r$ are L-collinear. Hence the lines of $Y_{0} \cap \Delta_{q}$ are precisely the lines of $\Delta_{q}\left(C_{q}\right) \cap \Delta_{q}(\{\langle q,(0,0,0,0 ; m)\rangle, \beta\})$, where $\beta$ is any plane through $\langle q,(0,0,0,0 ; m)\rangle$.

It is straightforward to calculate that, if a plane $\beta=\left[x_{1}^{\prime}, x_{2}^{\prime}, x_{3}^{\prime} ; k^{\prime}, l^{\prime}, m^{\prime}\right]$ contains the point $q$, then $x_{3}^{\prime}=\left(l-l^{\prime}\right) b^{\sigma}$ and $m^{\prime}=m-\left(l-l^{\prime}\right) b^{\sigma+1}$. By Proposition 3, such a plane belongs to $Y_{0}$ if and only $x_{3}^{\prime} \neq 0$ and $m^{\prime} \neq 0$.

Since $b \neq 0$, the equality $x_{3}^{\prime}=0$ is equivalent with $l=l^{\prime}$. This is equivalent with saying that the unique point $r$ of type 3 of $\beta$ can be written as $\left(x_{1}^{\prime \sigma}, 0 ; l\right)$. A plane $\gamma=\left[x_{1}^{\prime \prime}, x_{2}^{\prime \prime} ; k^{\prime \prime}, l^{\prime \prime}\right]$ of type VI contains $q$ if and only if $x_{1}^{\prime \prime}=\left(l-k^{\prime \prime}\right) b^{\sigma}, x_{2}^{\prime \prime}=0$ and $l^{\prime \prime}=m+\left(k^{\prime \prime}-l\right) b^{\sigma+1}$. Such a plane contains $r$ if and only if $k^{\prime \prime}=l$; indeed, this is necessary as the last coordinate of $r$, which is $l$, must be equal to the third coordinate of $\gamma$, which is $k^{\prime \prime}$, but it is also sufficient since $k^{\prime \prime}=l$ implies $x_{1}=0$. Hence, the unique plane of type VI through $q$ and $r$ has coordinates $[0,0 ; l, m]$, and it follows that a plane $\left[x_{1}^{\prime}, x_{2}^{\prime}, x_{3}^{\prime} ; k^{\prime}, l^{\prime}, m^{\prime}\right], x_{1}^{\prime}, x_{2}^{\prime}, x_{3}^{\prime} \in \mathbb{O}, k^{\prime}, l^{\prime}, m^{\prime} \in \mathbb{K}$, containing $q$ satisfies $x_{3}^{\prime} \neq 0$ if and only if it intersects the plane $[0,0 ; l, m]$ only in $q$, hence if and only if those two planes define opposite elements in the generalized quadrangle $\Delta_{q}$.

Likewise, with similar calculations, one shows that the condition $m^{\prime} \neq 0$ translates into $\left[x_{1}^{\prime}, x_{2}^{\prime}, x_{3}^{\prime} ; k^{\prime}, l^{\prime}, m^{\prime}\right]$ being opposite $\gamma^{\prime}=\left[m b^{-1}, 0 ; l-m b^{-\sigma-1}, 0\right]$ in $\Delta_{q}$. It is now easy to see that

$$
Y_{0} \cap \Delta_{q}=\Delta_{q}^{0}\left(C_{q}\right) \cap \Delta_{q}^{0}(\{\langle q,(0,0,0,0 ; m)\rangle, \gamma\}) \cap \Delta_{q}^{0}\left(\left\{\langle q,(0,0,0,0 ; m)\rangle, \gamma^{\prime}\right\}\right) .
$$


The result now follows from Corollary 1.

Using the Seifert-Van Kampen theorem, we now see that $Y_{1}$ is simply connected. We now add some lines to $Y_{1}$ and define

$$
Y_{2}=Y_{1} \cup\left\{L: L \text { is a line of type } L \text { containing a point of } Y_{1} \backslash Y_{0}\right\}
$$

In order to show that $\Delta_{L} \cap Y_{1}$ is connected for $L \in Y_{2} \backslash Y_{1}$, we only need to show that $L$ is contained in some plane of $Y_{0}$, as $\Delta_{L}$ is complete bipartite.

Proposition 5. Each line $L \in Y_{2} \backslash Y_{1}$ is contained in a plane of $Y_{0}$, and hence $Y_{2}$ is simply connected.

Proof. Let the line $L$ contain the point $q=\left(0, b, l b, d ; m+l b^{\sigma+1}\right)$, with $b \in \mathbb{O}^{\times}, d \in \mathbb{O}$ and $l, m \in \mathbb{K}$. Let the unique point of type 4 on $L$ have coordinates $\left(w_{1}, w_{2}, w_{3} ; i\right) \in$ $\mathrm{O}^{3} \times \mathbb{K}$. As in the previous proof, one calculates that a plane $\beta=\left[x_{1}^{\prime}, x_{2}^{\prime}, x_{3}^{\prime} ; k^{\prime}, l^{\prime}, m^{\prime}\right]$ contains $q$ if and only if

$$
\left\{\begin{array}{l}
x_{2}^{\prime}=d-x_{1}^{\prime \sigma} b^{\sigma}, \\
x_{3}^{\prime}=\left(l-l^{\prime}\right) b^{\sigma}, \\
m^{\prime}=m-\left(l-l^{\prime}\right) b^{\sigma+1} .
\end{array}\right.
$$

If we choose $l^{\prime} \notin\left\{l, l-m b^{-\sigma-1}\right\}$, which is always possible as $|\mathbb{K}|>2$, then $\beta$ contains $L$ and belongs to $Y_{0}$.

We now add some planes to $Y_{2}$ and define

$$
\begin{aligned}
Y_{3}=Y_{2} & \cup\left\{\left[x_{1}, x_{2}, x_{3} ; k, l, 0\right]: x_{3} \in \mathbb{O}^{\times}, x_{1}, x_{2} \in \mathbb{O}, k, l \in \mathbb{K}\right\} \\
& \cup\left\{\left[x_{1}, x_{2}, 0 ; k, l, m\right]: x_{1} \in \mathbb{O}, x_{2} \in \mathbb{O}^{\times}, k, l \in \mathbb{K}, m \in \mathbb{K}^{\times}\right\} .
\end{aligned}
$$

Proposition 6. Let $\alpha \in Y_{3} \backslash Y_{2}$. Then $\Delta_{\alpha} \cap Y_{2}$ is connected, and hence $Y_{3}$ is simply connected.

Proof. Let $A$ be the set of points in $\Delta_{\alpha} \cap Y_{0}$, let $B$ be the set of points in $\Delta_{\alpha} \cap\left(Y_{1} \backslash Y_{0}\right)$, and let $W$ be the set of lines in $\Delta_{\alpha} \cap Y_{2}$. Note that all points of $A$ have a nonzero first 
coordinate (indeed, every such point is L-collinear with $p$ and distinct from $p$ since either $x_{2} \neq 0$ or $x_{3} \neq 0$ ) and all points in $B$ have as first coordinate 0 . So $A \cap B=\varnothing$. We first show that $A \neq \varnothing \neq B$.

- $\alpha=\left[x_{1}, x_{2}, x_{3} ; k, l, 0\right]$, with $x_{3} \in \mathbb{O}^{\times}, x_{1}, x_{2} \in \mathbb{O}, k, l \in \mathbb{K}$. The point of type 3 in $\alpha$ is $\left(x_{1}^{\sigma}, x_{3} ; l\right)$, which is never collinear with $p$, since $x_{3} \neq 0$. Hence $\alpha^{\perp p}$ is a line of type $\mathrm{L}$, and so $A \neq \varnothing$ in this case. Also, $p_{\alpha, 1}\left(0, x_{3}^{\sigma}\right)$ is K-collinear with $\left(0,0,0,0 ; x_{3}^{\sigma}\right)$ since both points have 0 as first coordinate and lie in the common plane $\left[x_{3}^{-1} x_{2}+x_{1}, 0,0 ; 0,1+l, x_{3}^{\sigma+1}\right]$. So $B \neq \varnothing$.

- $\alpha=\left[x_{1}, x_{2}, 0 ; k, l, m\right]$, with $x_{1} \in \mathrm{O}, x_{2} \in \mathrm{O}^{\times}, k, l \in \mathbb{K}, m \in \mathbb{K}^{\times}$. The point of type 3 in $\alpha$ is $\left(x_{1}^{\sigma}, 0 ; l\right)$, which is always collinear with $p$, and we also have that neither $p_{\alpha, 1}(0,0)=\left(0,0,0, x_{2}^{\sigma} ; m\right)$ nor $p_{\alpha, 2}(0)=\left(0, x_{1}, x_{2} ; k\right)$ is collinear with $p$ (all this because $x_{2} \neq 0$ and a plane of type VII or VIII containing $p$ must have second coordinate 0 ). Hence every point in $\alpha$ distinct from $p_{\alpha, 3}$ collinear with $p$ has type 5 and has nonzero first coordinate, hence belongs to $Y_{0}$ and to $A$. Also, the point $p_{\alpha, 1}(0, b)=\left(0, b, l b, x_{2}^{\sigma}+x_{1}^{\sigma} b ; m+l b^{\sigma+1}\right)$ is K-collinear with and distinct from $(0,0,0,0 ; m)$, for every $b \in \mathbb{O}^{\times}$, see the first paragraph of the proof of Proposition 4 . Hence $B \neq \varnothing$.

Now, if a line $L$ in $\alpha$ belongs to $Y_{0}$, then it contains a point of $A$. Likewise, if a line $L$ in $\alpha$ belongs to $Y_{2} \backslash Y_{0}$, then it contains a point of $B$. Moreover, every line of type $L$ through any point of $B$ is contained in $Y_{2}$. Noting that all lines connecting a point of $A$ with a point of $B$ have type $\mathrm{L}$, we immediately deduce that $A \cap B \cap W$ is connected.

Note that a plane $\left[x_{1}, x_{2}, x_{3} ; k, l, m\right], x_{1}, x_{2}, x_{3} \in \mathbb{O}, k, l, m \in \mathbb{K}$, belongs to $Y \backslash Y_{3}$ if and only if $x_{3}=0$ and either $x_{2}=0$ and $m \neq 0$, or $x_{2} \neq 0$ and $m=0$. These will be added in two distinct steps $\left(Y_{5}\right.$ and $Y_{7}$, respectively).

Now we again add some points.

$Y_{4}=Y_{0} \cup \bigcup_{m \in \mathbb{K}^{\times}}\{q \in Y: q$ is a point L-collinear with and distinct from $(0,0,0,0 ; m)\}$. 
We first establish the general form of a point in $Y_{4} \backslash Y_{1}$. Firstly, an arbitrary plane $\alpha$ of type VIII containing the point $p_{\alpha, 1}(0,0)=(0,0,0,0 ; m)$ has coordinates $\left[x_{1}, 0,0 ; k, l, m\right], x_{1} \in \mathrm{O}, k, l \in \mathbb{K}$. An arbitrary point in that plane L-collinear with and distinct from $(0,0,0,0 ; m)$ is just an arbitrary point in $\alpha$ with first coordinate, say $a \in \mathrm{O}$, unequal 0 , i.e.,

$$
q=\left(a, b, l b+x_{1} a, k a+x_{1}^{\sigma} b ; m+k a^{\sigma+1}+l b^{\sigma+1}+\left(a^{\sigma} x_{1}^{\sigma}\right) b+b^{\sigma}\left(x_{1} a\right)\right), a \neq 0 .
$$

Proposition 7. Let $q \in Y_{4} \backslash Y_{3}$. Then $\Delta_{q} \cap Y_{3}$ is connected and hence $Y_{4}$ is simply connected.

Proof. Put $q=\left(a, b, l b+x_{1} a, k a+x_{1}^{\sigma} b ; m+k a^{\sigma+1}+l b^{\sigma+1}+\left(a^{\sigma} x_{1}^{\sigma}\right) b+b^{\sigma}\left(x_{1} a\right)\right)$, as above and put $r=\left(0,0,0, m a^{-\sigma} ; 0\right)$. It is clear that every plane of type VIII incident with $r$ must have the third and last coordinate equal to 0 . Let $\beta=\left[x_{1}^{\prime}, x_{2}^{\prime}, 0 ; k^{\prime}, l^{\prime}, 0\right]$, with $x_{1}^{\prime}, x_{2}^{\prime} \in \mathbb{O}$ and $k^{\prime}, l^{\prime} \in \mathbb{K}$ arbitrary, and suppose $q \in \beta$. The description of planes of type VIII implies

$$
\left\{\begin{aligned}
l b+x_{1} a & =l^{\prime} b+x_{1}^{\prime} a, \\
k a+x_{1}^{\sigma} b & =x_{2}^{\prime \sigma}+k^{\prime} a+x_{1}^{\prime \sigma} b, \\
m+k a^{\sigma+1}+l b^{\sigma+1}+\left(a^{\sigma} x_{1}^{\sigma}\right) b+b^{\sigma}\left(x_{1} a\right) & =x_{2}^{\prime} a+a^{\sigma} x_{2}^{\prime \sigma}+k^{\prime} a^{\sigma+1}+l^{\prime} b^{\sigma+1}+\left(a^{\sigma} x_{1}^{\prime \sigma}\right) b+b^{\sigma}\left(x_{1}^{\prime} a\right) .
\end{aligned}\right.
$$

Putting $L=l-l^{\prime}, K=k-k^{\prime}$ and $X_{1}=x_{1}-x_{1}^{\prime}$, we can rewrite this as

$$
\left\{\begin{aligned}
L b+X_{1} a & =0, \\
K a+X_{1}^{\sigma} b & =x_{2}^{\prime \sigma}, \\
m+K a^{\sigma+1}+L b^{\sigma+1}+\left(a^{\sigma} X_{1}^{\sigma}\right) b+b^{\sigma}\left(X_{1} a\right) & =x_{2}^{\prime} a+a^{\sigma} x_{2}^{\prime \sigma} .
\end{aligned}\right.
$$

Remembering $K, L \in \mathbb{K}$, we deduce from the first equation that $X_{1}$ belongs to the skew field generated by $a$ and $b$; then the second equation says that also $x_{2}^{\prime}$ belongs to this skew field. Hence all elements in this system of equations belong to a common skew field, and so we may remove the parentheses. It is now an elementary exercise to eliminate $L$ and $K$ from the first two equations, and then 
also $X_{1}$ disappears in the last equation, giving $m=x_{2}^{\prime} a$. Noting that $\left(0,0,0, x_{2}^{\prime \sigma} ; 0\right)$ always belongs to $\left[x_{1}^{\prime}, x_{2}^{\prime}, 0 ; k^{\prime}, l^{\prime}, 0\right]$, we conclude that $r \in \beta$. Hence a plane of type VIII containing $q$ has the form $\left[x_{1}^{\prime}, x_{2}^{\prime}, 0 ; k^{\prime}, l^{\prime}, 0\right], x_{1}^{\prime}, x_{2}^{\prime} \in \mathbb{O}, k^{\prime}, l^{\prime} \in \mathbb{K}$, if and only if it contains $r$.

Now put $s=(0,0,0,0 ; m)$. It is clear that every plane of type VIII incident with $s$ must have second and third coordinate equal to 0 . Conversely, let $\gamma$ be an arbitrary plane with coordinates $\left[x_{1}^{\prime \prime}, 0,0 ; k^{\prime \prime}, l^{\prime \prime}, m^{\prime \prime}\right]$ and suppose $q \in \gamma$. Note that $\left(0,0,0,0 ; m^{\prime \prime}\right) \in \gamma$. But then Lemma 1 implies $m=m^{\prime \prime}$, yielding $s \in \gamma$. Hence a plane of type VIII containing $q$ has the form $\left[x_{1}^{\prime \prime}, 0,0 ; k^{\prime \prime}, l^{\prime \prime}, m^{\prime \prime}\right], x_{1}^{\prime \prime} \in \mathrm{O}, k^{\prime \prime}, l^{\prime \prime}, m^{\prime \prime} \in \mathbb{K}$, if and only if it contains $s$. We conclude that the planes of $Y_{3}$ in $\Delta_{q}$ are precisely the planes of $\Delta_{q}^{0}\left(C_{q}\right) \cap \Delta_{q}^{0}(\langle r, q\rangle) \cap \Delta_{q}^{0}(\langle s, q\rangle)$.

Noting that $p$ is not collinear with $q$, a line $R$ of type 5 through $q$ is not contained in $Y_{0}$ if and only if its unique point of type 4 is collinear with $p$, or its unique point of type 5 with first coordinate 0 is collinear with $p$. Now, by Lemma 1, the point of type 4 on $R$ is collinear with $p$ if and only if it is collinear with $s$ if and only if $\langle q, s\rangle$ is collinear with $R$ in $\Delta_{q}$. Now let $u$ be a point of type 5 with first coordinate 0 . Suppose first that $u$ is collinear with $p$. Since $u$ is not L-collinear with $p$, one verifies (see [7]) that the line $\langle u, p\rangle$ is contained in a unique plane of type either V or VI, of the form $[0,0 ; 0]$ or $[0,0 ; k, 0], k \in \mathbb{K}$ respectively. But such a plane automatically contains $r$. Conversely, if $u$ is collinear with $r$, then the same argument implies that $p$ is collinear with $u$. Hence we have shown that the lines of $Y_{0}$ in $\Delta_{q}$ are precisely those of $\Delta_{q}^{0}\left(C_{q}\right) \cap \Delta_{q}^{0}(\langle r, q\rangle) \cap \Delta_{q}^{0}(\langle s, q\rangle)$. By Corollary 1 , this is connected. Proposition 5 implies that $\Delta_{q} \cap Y_{3}$ is connected.

We now add half of the missing planes to $Y_{4}$ and define

$$
Y_{5}=Y_{4} \cup\left\{\left[x_{1}, 0,0 ; k, l, m\right]: x_{1} \in \mathbb{O}, k, l \in \mathbb{K}, m \in \mathbb{K}^{\times}\right\}
$$

Proposition 8. Let $\alpha \in Y_{5} \backslash Y_{4}$. Then $\Delta_{\alpha} \cap Y_{4}$ is connected and hence $Y_{5}$ is simply connected.

Proof. Let $\alpha=\left[x_{1}, 0,0 ; k, l, m\right], x_{1} \in \mathbb{O}, k, l \in \mathbb{K}, m \in \mathbb{K}^{\times}$. We note that $(0,0,0,0 ; m) \in$ 
$\alpha$, hence by the definition of $Y_{1}$ and $Y_{4}$, all other points of type 5 of $\alpha$ are contained in $Y_{4}$. By the definition of $Y_{2}$, all lines in $\alpha$ of type $\mathrm{L}$ containing a point $p_{\alpha, 1}(0, b)$, $b \in \mathbb{O}^{\times}$, belong to $Y_{4}$. This implies easily that $\Delta_{\alpha} \cap Y_{4}$ is connected (in fact, it suffices to find two lines through each point of $\alpha$ that belongs to $Y_{4}$, but here we found infinitely many).

So far, $Y_{5}$ contains all lines of type $\mathrm{L}$ all of whose points are collinear with $p$. It also contains all lines of type L with a unique point $q$ collinear with $p$ such that $q$ has type 5 and is L-collinear with $p$, or such that $q$ has type 4 and the line contains a point $\mathrm{K}$-collinear with some $(0,0,0,0 ; m), m \in \mathbb{K}^{\times}$. Since all lines of type $\mathrm{L}$ contain some point collinear with $(0,0,0,0 ; m)$, for all $m \in \mathbb{O}$, it is natural to first concentrate on special cases of that collinearity.

Also, there are still lines in $Y$ not incident with any point of $Y_{5}$. We cannot add those lines, as such a line $M$ would give rise to non-connected $\Delta_{M} \cap Y_{5}$. For instance, one checks that the line $T$ of the plane $\alpha=[0,1,1 ; 0,0,0]$ containing the points $p_{\alpha, 2}(-1)$ and $p_{\alpha, 1}(0, x)$, with $x \in \mathbb{O} \backslash \mathbb{K}$, does not contain any point of type 5 collinear with any point $(0,0,0,0 ; m), m \in \mathbb{K}$.

Hence we now add some specific lines to $Y_{5}$ and define $Y_{6}$ as follows.

$Y_{6}=Y_{5} \cup\left\{\left\langle q, q^{\prime}\right\rangle: q=(a, b, c, d ; k) \in Y_{4}, b \neq 0\right.$ and $\left.q \perp q^{\prime}=\left(a^{\prime}, 0, c^{\prime}, d^{\prime} ; k^{\prime}\right), a^{\prime} \notin\{0, a\}\right\}$.

By definition, every line $R \in Y_{6} \backslash Y_{5}$ contains at least one point of $Y_{4}$; so to prove connectivity of $\Delta_{R} \cap Y_{5}$, it suffices to prove that there is at least one plane of $Y_{5}$ incident with $R$.

Proposition 9. There is at least one plane of $Y_{3}$ containing any line $R$ of $Y_{6} \backslash Y_{5}$. Consequently, $\Delta_{R} \cap Y_{5}$ is connected and hence $Y_{6}$ is simply connected.

Proof. Every line of type L is incident with infinitely many planes of type VIII, and exactly one of type VII. Suppose two planes $\alpha_{1}, \alpha_{2}$ of type VIII meet in $R$ and let $\alpha_{i}=\left[x_{i}, y_{i}, 0 ; k_{i}, l_{i}, 0\right], x_{i}, y_{i} \in \mathrm{O}, y_{i} \neq 0, k_{i}, l_{i} \in \mathbb{K}, i=1,2$.

Let $R=\left\langle q, q^{\prime}\right\rangle$ with $q=(a, b, c, d ; k) \in Y_{4}, b \neq 0, q \perp q^{\prime}=\left(a^{\prime}, 0, c^{\prime}, d^{\prime} ; k^{\prime}\right), a^{\prime} \notin$ $\{0, a\}$. Let $r=\left(w_{1}, w_{2}, w_{3} ; i\right)$ be the unique point of type 4 in $R$. Note that this implies, 
by the definition of lines in $\Delta$, that $b-w_{1} a=0-w_{1} a^{\prime}$, hence $w_{1}=b\left(a-a^{\prime}\right)^{-1} \neq 0$. Since $r \in \alpha_{i}, i=1,2$, we easily deduce

$$
\left\{\begin{array}{l}
x_{1}-x_{2}=-\left(l_{1}-l_{2}\right) w_{1} \\
k_{1}-k_{2}=\left(l_{1}-l_{2}\right) w_{1}^{\sigma+1} .
\end{array}\right.
$$

Since $a^{\prime} \neq 0$ and $w_{1} \neq 0, R$ contains a point $p_{\alpha_{i}, 1}(0, e)$, with $e \neq 0, i=1,2$. This easily yields, by comparing the third coordinate of $p_{\alpha_{1}, 1}(0, e)=p_{\alpha_{2}, 1}(0, e), l_{1} b=l_{2} b$, hence $l_{1}=l_{2}$, and by the above equalities, $x_{1}=x_{2}$ and $k_{1}=k_{2}$. From the fourth coordinate of $p_{\alpha_{1}, 1}(0, e)=p_{\alpha_{2}, 1}(0, e)$ follows $y_{1}=y_{2}$, hence $\alpha_{1}=\alpha_{2}$. It follows that there is at most one plane of type VIII not in $Y_{5}$ that contains $R$. The proposition is proved.

Now we add all remaining planes to $Y_{6}$.

$$
Y_{7}=Y_{6} \cup\left\{\left[x_{1}, x_{2}, 0 ; k, l, 0\right]: x_{1} \in \mathbb{O}, k, l \in \mathbb{K}, x_{2} \in \mathbb{O}^{\times}\right\} .
$$

Proposition 10. For any plane $\alpha \in Y_{7} \backslash Y_{6}$, we have that $\Delta_{\alpha} \cap Y_{6}$ is connected and hence $Y_{7}$ is simply connected.

Proof. Put $\alpha=\left[x_{1}, x_{2}, 0 ; k, l, 0\right]: x_{1} \in \mathbb{O}, k, l \in \mathbb{K}, x_{2} \in \mathbb{O}^{\times}$. Let us determine the points of $Y_{6}$ in $\alpha$. Note first that $p_{\alpha, 3}=\left(x_{1}^{\sigma}, 0 ; l\right)=p_{\beta, 3}$, with $\beta=\left[x_{1}, 0,0 ; 0, l, 0\right] \ni p$. Also, $p_{\alpha, 1}(0,0)=\left(0,0,0, x_{2}^{\sigma} ; 0\right)=p_{\gamma, 1}\left(0, x_{2}^{\sigma}\right) \ni p$, where $\gamma=[0,0 ; 0]$. Hence the line $\alpha^{\perp p}$ has type $\mathrm{K}$ and all of its points of type 5 have 0 as first coordinate and so are not L-collinear with $p$. Consequently, there are no points of $Y_{0}$ in $\alpha$.

By Lemma 1 , every point $r_{m}:=(0,0,0,0 ; m), m \in \mathbb{K}$, is collinear with $p_{\alpha, 3}$. Hence all points of type 5 collinear with $r_{m}$ have the same first coordinate (distinct from 0 by Lemma 1 again), and so there is one with second coordinate 0 , say $p_{\alpha, 1}(a, 0)$. Since $a \neq 0, p_{\alpha, 1}(a, 0)$ cannot be K-collinear with $r_{m}$. So, no point of $\alpha$ belongs to $Y_{1}$. The calculation in the first part of the proof of Proposition 7 shows that $p_{\alpha, 1}(a, 0)$ is L-collinear to $r_{m}$ if and only if $m=x_{2} a$, hence $a$ ranges over all $\mathbb{K}$-multiples of $x_{2}^{-1}$. Since every line of type $\mathrm{L}$ in $\alpha$ contains a point of type 5 with first coordinate $x_{2}^{-1}$, 
it suffices to show that two arbitrary points of $Y_{4}$ in $\alpha$ can be joined by a path of $Y_{6}$ in $\alpha$. By the definition of lines in $Y_{6} \backslash Y_{5}$, every point of $Y_{4}$ in $\alpha$ is connected by a single line to $p_{\alpha, 1}\left(x_{2}^{-1}, 0\right)$, except for the points $p_{\alpha, 1}\left(x_{2}^{-1}, b\right), b \in \mathrm{O}^{\times}$, and the points $p_{\alpha, 1}(a, 0), a \neq x_{2}^{-1}$. Every point $p_{\alpha, 1}(a, 0), a \neq x_{2}^{-1}$ is connected with every point $p_{\alpha, 1}\left(x_{2}^{-1}, b\right), b \in \mathrm{O}^{\times}$, so we have at most two connected components. Now, picking two distinct $m, m^{\prime} \in \mathbb{K} \backslash\{0,1\}$, we see these two sets are connected since the point $p_{\alpha, 1}\left(m x_{2}^{-1}, 1\right)$ is connected with $p_{\alpha, 1}\left(m^{\prime} x_{2}^{-1}, 0\right)$.

We now add all the lines having a point in $Y_{7}$ (and we know that there are still others, see the example of the line $T$ above).

$$
Y_{8}=Y_{7} \cup\left\{M: M \text { is a line of type } \mathrm{L} \text { containing a point in } Y_{4}\right\}
$$

Since every line in $Y_{8} \backslash Y_{7}$ is by definition incident with a point of $Y_{7}$, and since $Y_{7}$ contains all planes of type VIII, and every line of type $L$ is inside a plane of type VIII, we know that $\Delta_{M} \cap Y_{7}$ is connected, for $M \in Y_{8} \backslash Y_{7}$, and hence $Y_{8}$ is simply connected.

We now add all remaining points to $Y_{8}$ and define

$$
Y_{9}=Y_{8} \cup\{q: q \text { is a point of type } 5\}
$$

Proposition 11. For any point $q \in Y_{9} \backslash Y_{8}$, we have that $\Delta_{q} \cap Y_{8}$ is connected and hence $Y_{9}$ is simply connected.

Proof. First note that $Y_{8}$ contains all planes of $Y$, hence if we consider a line $M \ni q$ of $Y_{8}$, then all but exactly one planes of $\Delta_{q}$ incident with $M$ belong to $\Delta_{q} \cap Y_{8}$. Now let $\alpha=\left[x_{1}, x_{2}, x_{3} ; k, l, m\right], x_{1}, x_{2}, x_{3} \in \mathbb{O}, k, l, m \in \mathbb{K}$, be a plane through $q$. We show that at most two lines of $\alpha$ through $q$ do not belong to $Y_{8}$. It will then follow from Proposition 1 that $\Delta_{q} \cap Y_{8}$ is connected.

Suppose first that $x_{3} \neq 0$. Then there is a unique point $r=p_{\alpha, 2}\left(-x_{3}^{-1} x_{2}\right)$ of type 4 with third coordinate 0 . Let $M$ be a line in $\alpha$ through $q$ distinct from (1) the line of type $\mathrm{K}$ through $q$, and (2) the line $\langle q, r\rangle$. Then $M$ has type $\mathrm{L}$ and the point $r_{M}$ on $M$ 
of type 4 has third coordinate distinct from 0 . It is easily seen that this implies that $r_{M}$ is not collinear with $p$, hence, by Lemma $1, r_{M}$ is not collinear with any point $\left(0,0,0,0 ; m^{\prime}\right)$, for any $m^{\prime} \in \mathbb{K}$. Hence, again using Lemma 1 , all points $s_{m^{\prime}}$ collinear with $\left(0,0,0,0 ; m^{\prime}\right), m^{\prime} \in \mathbb{K}$, and incident with $M$ are distinct, and so at least one has first coordinate nonzero, implying that it is L-collinear with the corresponding point $\left(0,0,0,0 ; m^{\prime}\right)$. This, in turn, implies that $M$ belongs to $Y_{8}$.

Hence we may assume that $x_{3}=0$. If $x_{2} \neq 0$, then no point of type 4 in $\alpha$ has third coordinate equal to 0 , and so the argument of the previous paragraph implies that only the line of type $\mathrm{K}$ through $q$ in $\alpha$ does not belong to $Y_{8}$, but all other lines in $\alpha$ through $q$ do.

Hence we may assume that $x_{2}=x_{3}=0$. In this case $(0,0,0,0 ; m) \in \alpha$, and then it follows from the definitions of $Y_{1}, Y_{2}, Y_{4}$ and $Y_{8}$ that all lines of type L through $q$ in $\alpha$ belong to $Y_{8}$.

Now that we have all points and planes of $Y$ in $Y_{9}$, we can add all remaining lines and define $Y_{10}=Y$. Clearly, for any line $M \in Y_{10}$, the geometry $\Delta_{M} \cap Y$ is connected and, once again, the Seifert-Van Kampen theorem implies that $Y$ is simply connected. This completes the proof of Theorem 1 for the nonembeddable thick polar spaces. 


\section{Chapter 3}

\section{Group-theoretic applications}

\subsection{First results}

We will now apply our knowledge that $\Delta^{0}(C)$ is simply connected when $\Delta$ is a nonembeddable polar space to express certain groups as amalgams. The following result of Soulé [12] provides the basic tool.

Proposition 12. Suppose a group $G$ acts on a simplicial complex $T$ with simplicial fundamental domain $T^{\prime}$. Denote by $E^{\prime}$ the vertex set of $T^{\prime}$ and by $R(T), R\left(T^{\prime}\right)$ the geometric realizations of $T$ and $T^{\prime}$. If $R\left(T^{\prime}\right)$ is connected and $R(T)$ is simply connected, then $G$ is the free product of the stabilizers $G_{v}\left(v \in E^{\prime}\right)$ amalgamated with respect to their intersections.

Already in [12], Proposition 12 is used to express a group $G$ with BN-pair (of rank at least 3 if spherical) as an amalgam of its maximal standard parabolic subgroups, i.e. maximal subgroups containing $B$. The proof uses the full action of $G$ on the associated building - simply connected by the Solomon-Tits theorem—with a chamber as fundamental domain.

Our recently submitted joint paper [9] contains sharp bounds, improving on those of Abramenko [2], that characterize when $\Delta^{0}(C)$ is simply connected for finite polar spaces. The proof, due to Max Horn, is via computer calculations. Combining this result with Theorem 1 and Abramenko's original results about $\Delta^{0}(C)$ in the rank-3 case, we obtain the following 
Theorem 2. Let $G$ be a group with spherical BN-pair of irreducible rank 3. Assume that every panel of the associated building $\Delta=\Delta(G, B)$ is contained in at least 6 chambers. Let $D$ be a chamber opposite $C=B \in \Delta(G, B)$. Then $B$ is the free product of the stabilizers $B_{v}$, where $v$ ranges over the vertices of $D$, amalgamated with respect to their intersections.

Proof. If $\Delta$ is associated to a nonembeddable polar space, then $\Delta^{0}(C)$ is simply connected by Theorem 1 . Otherwise, $\Delta^{0}(C)$ is simply connected according to $[2$, Theorem $B]$ in case $\Delta$ has type $A_{3}$, or $\left[9\right.$, Corollary 2.3] in case $\Delta$ has type $C_{3}$. B acts on $\Delta^{0}(C)$ with the geometric simplex $\bar{D}$ as a simplicial fundamental domain, and the result follows from Proposition 12.

Remark. The theorem remains true for BN-pairs of rank greater than 3. This follows from Corollary 2 below, viewing the spherical building as a twin building.

\subsection{An application involving twin buildings}

Let $\Delta=\left(\Delta_{+}, \Delta_{-}, \delta^{*}\right)$ be a thick twin building of type $(W, S)$ and rank at least 3 . Fix a pair of opposite chambers $C_{+} \in \Delta_{+}, C_{-} \in \Delta_{-}$. As previously mentioned, we denote by $\Delta^{0}\left(C_{-}\right)$the subcomplex of $\Delta_{+}$generated by the chambers opposite $C_{-}$. We now investigate the simple connectivity of $\Delta^{0}\left(C_{-}\right)$by defining an appropriate filtration.

The numerical codistance function $d^{*}=\ell \circ \delta^{*}$ between pairs of chambers extends to pairs of simplices via

$$
d^{*}(a, b)=\min \left\{d^{*}(x, y): x \text { and } y \text { are chambers, } a \leq x, b \leq y\right\}
$$

and therefore $\Delta^{0}\left(C_{-}\right)=\left\{x_{+} \in \Delta_{+}: d^{*}\left(C_{-}, x_{+}\right)=0\right\}$. More generally we define for every $j \in \mathbb{N}_{0}$ the subcomplex

$$
\Delta_{j}=\left\{x_{+} \in \Delta_{+}: d^{*}\left(C_{-}, x_{+}\right) \leq j\right\}
$$

of $\Delta_{+}$. 
For a chamber $D_{+} \in \Delta_{+}$we define its restriction with respect to $C_{-}$, denoted $R_{C_{-}}\left(D_{+}\right)$, as its face of cotype $\{s \in S: \ell(w s)>\ell(w)\}$, where $w=\delta^{*}\left(C_{-}, D_{+}\right)$.

Lemma 2. For any face $b_{+} \leq D_{+}, d^{*}\left(C_{-}, b_{+}\right)=d^{*}\left(C_{-}, D_{+}\right)$if and only if $R_{C_{-}}\left(D_{+}\right) \leq b_{+}$.

Proof. Denote by $C\left(b_{+}\right)$the set of chambers of $\Delta_{+}$that contain $b_{+}$. We have $\delta^{*}\left(\left\{C_{-}\right\} \times C\left(b_{+}\right)\right)=w W_{K}$ where $K$ is the cotype of $b_{+}[3$, Lemma 5.148]. The coset $w W_{K}$ contains a unique representative $w_{0}$ of minimal length, characterized by the property that $\ell\left(w_{0} s\right)=\ell\left(w_{0}\right)+1$ for all $s \in K$ [3, Proposition 2.20]. Thus

$$
d^{*}\left(C_{-}, b_{+}\right)=d^{*}\left(C_{-}, D_{+}\right) \Leftrightarrow w=w_{0} \Leftrightarrow K \subseteq\{s \in S: \ell(w s)>\ell(w)\} .
$$

We now make several definitions related to the filtration of $\Delta_{+}$. Here $j \in \mathbb{N}_{0}$.

$$
\begin{aligned}
R_{j+1} & :=\left\{R_{C_{-}}\left(D_{+}\right): D_{+} \in \Delta_{j+1} \backslash \Delta_{j}, D_{+} \text {is a chamber }\right\} \\
b_{+} \in R_{j+1} \sim S\left(b_{+}\right) & :=\left\{\gamma_{+} \in \Delta_{+}: \gamma_{+} \cup b_{+} \in \Delta_{j+1}\right\}=\mathrm{st}_{\Delta_{j+1}}\left(b_{+}\right) \\
T^{\prime}\left(b_{+}\right) & :=\left\{\gamma_{+} \in S\left(b_{+}\right): \gamma_{+} \cap b_{+}=\varnothing\right\}=1 \mathrm{k}_{\Delta_{j+1}}\left(b_{+}\right) \\
T\left(b_{+}\right) & :=S\left(b_{+}\right) \cap \Delta_{j}
\end{aligned}
$$

\section{Lemma 3.}

1. $\Delta_{j+1}=\Delta_{j} \cup \bigcup_{b_{+} \in R_{j+1}} S\left(b_{+}\right)$

2. $S\left(b_{+}\right) \cap S\left(b_{+}^{\prime}\right) \subseteq \Delta_{j}$ for all $b_{+} \neq b_{+}^{\prime} \in R_{j+1}$

3. $T\left(b_{+}\right)=\partial b_{+} * T^{\prime}\left(b_{+}\right)$

Proof.

1. The inclusion " $\supseteq$ " is clear, since by definition $S\left(b_{+}\right) \subseteq \Delta_{j+1}$ for any $b_{+} \in R_{j+1}$. 
As for " $\subseteq$ ", we have

$$
\begin{aligned}
\Delta_{j+1} \backslash \Delta_{j}= & \left\{x_{+} \in \Delta_{+}: d^{*}\left(C_{-}, x_{+}\right)=j+1\right\} \\
= & \left\{x_{+} \in \Delta_{+}: x_{+} \leq D_{+} \text {for some chamber } D_{+} \in \Delta_{j+1} \backslash \Delta_{j}\right. \\
& \text { with } \left.d^{*}\left(C_{-}, x_{+}\right)=d^{*}\left(C_{-}, D_{+}\right)\right\} \\
= & \bigcup_{\substack{D_{+} \in \Delta_{j+1} \\
D_{+} \text {chamber }}}\left\{x_{+} \leq D_{+}: R_{C_{-}}\left(D_{+}\right) \leq x_{+}\right\} \\
\subseteq & \bigcup_{b_{+} \in R_{j+1}} S\left(b_{+}\right)
\end{aligned}
$$

according to Lemma 2.

2. Write $b_{+}=R_{C_{-}}\left(D_{+}\right)$and $b_{+}^{\prime}=R_{C_{-}}\left(D_{+}^{\prime}\right)$. If $D_{+} \cap D_{+}^{\prime} \notin \Delta_{j}$ then $D_{+} \cap D_{+}^{\prime}$ contains a smallest face not in $\Delta_{j}$, namely $R_{C_{-}}\left(D_{+}\right)=R_{C_{-}}\left(D_{+}^{\prime}\right)$, according to Lemma 2. This contradicts the assumption that $b_{+} \neq b_{+}^{\prime}$.

3. This follows from the fact that $T\left(b_{+}\right)=\left\{\gamma_{+} \in S\left(b_{+}\right): \gamma_{+} \geq b_{+}\right\}$, itself a consequence of Lemma 2.

Let $b_{+} \in R_{j+1}$ and choose a twin apartment $\Sigma=\left(\Sigma_{+}, \Sigma_{-}\right)$such that $C_{-} \in \Sigma_{-}$ and $b_{+} \in \Sigma_{+}$. Set $b_{-}=\operatorname{op}_{\Sigma}\left(b_{+}\right) \in \Sigma_{-}$and $P_{-}=\operatorname{proj}_{b_{-}} C_{-}$. Then $\Delta^{\prime}=\left(\Delta_{b_{+}}, \Delta_{b_{-}}, \delta^{*} \mid\right)$ is a twin building of lower rank than $\Delta$.

As explained in the proof of Lemma 2,

$$
d^{*}\left(C_{-}, x_{+}\right)=d^{*}\left(C_{-}, b_{+}\right) \Leftrightarrow \delta^{*}\left(C_{-}, x_{+}\right)=w_{0}
$$

for any $x_{+} \in C\left(b_{+}\right)$. At the same time, there is a one-to-one correspondence

$$
\mathcal{C}\left(T^{\prime}\left(b_{+}\right)\right) \leftrightarrow\left\{x_{+} \in C\left(b_{+}\right): d^{*}\left(C_{-}, x_{+}\right)=d^{*}\left(C_{-}, b_{+}\right)\right\}
$$

Lemma 4. $T^{\prime}\left(b_{+}\right)$is isomorphic to $\Delta_{0^{\prime}}^{\prime}$, the first term of the filtration of $\Delta_{b_{+}}$with respect to $P_{-} \in \Delta_{b_{-}}$. 
Proof. First let us give a characterization of $P_{-}=\operatorname{proj}_{b_{-}} C_{-}$. As $C_{-}, b_{-}, b_{+} \in \Sigma$, we have $\delta_{-}\left(\left\{C_{-}\right\} \times C\left(b_{-}\right)\right)=\delta^{*}\left(\left\{C_{-}\right\} \times C\left(b_{+}\right)\right)=w_{0} W_{K}$. So the chamber $P_{-} \in \mathcal{C}\left(b_{-}\right)$ minimizing $d\left(\left\{C_{-}\right\} \times C\left(b_{-}\right)\right)$is the (unique) chamber of $\Sigma_{-}$with $\delta_{-}\left(C_{-}, P_{-}\right)=w_{0}$.

Now we prove the lemma. In light of (3.1) and (3.2), it suffices to establish the following: for any chamber $x_{+} \in C\left(b_{+}\right)$,

$$
\delta^{*}\left(C_{-}, x_{+}\right)=w_{0} \Leftrightarrow d^{*}\left(P_{-}, x_{+}\right)=0 .
$$

“ $\Rightarrow "$ : As $\delta^{*}\left(C_{-}, x_{+}\right)=\delta_{-}\left(C_{-}, P_{-}\right), x_{+}$op $P_{-}$by [3, Corollary 5.141].

" $\Leftarrow$ ": According to [3, Corollary 5.140], $\delta^{*}\left(C_{-}, x_{+}\right)$can be written as some subword of a reduced decomposition of $\delta_{-}\left(C_{-}, P_{-}\right)=w_{0}$. At the same time, $\delta^{*}\left(C_{-}, x_{+}\right) \in w_{0} W_{K}, \operatorname{so} d\left(C_{-}, x_{+}\right) \geq \ell\left(w_{0}\right)$. Together these facts imply that $\delta^{*}\left(C_{-}, x_{+}\right)=$ $w_{0}$.

We are now ready to reduce the study of $\Delta^{0}\left(C_{-}\right)$to criteria involving small links in $\Delta$. By 3-spherical we mean that every rank-3 link is spherical.

Proposition 13. Suppose that the twin building $\Delta$ is 3-spherical and satisfies

1. for every rank-2 link $\theta$ in $\Delta$ and any chamber $x \in \theta, \theta^{0}(x)$ is connected; and

2. for every rank-3 link $\theta$ in $\Delta$ and any chamber $x \in \theta, \theta^{0}(x)$ is 1-connected.

Then $\Delta^{0}\left(C_{-}\right)$is 1-connected.

Remark. An equivalent statement, proven using a combinatorial argument involving chamber systems, was given in [8, Theorem 1.1].

Proof. As a consequence of the first hypothesis, $\Delta^{0}\left(C_{-}\right)$is connected, even gallery connected [11, Theorem 1.5]. We now show that $\pi_{1}\left(\Delta^{0}\left(C_{-}\right)\right)$is trivial, proceeding by induction on the rank of $\Delta$.

The base case $\mathrm{rk} \Delta=3$ is immediate from the second hypothesis. For higher rank, we use Lemma 3. By 3., the cone over $T\left(b_{+}\right)$is $\partial b_{+} * T^{\prime}\left(b_{+}\right) *$ point, which is homeomorphic to $b_{+} * T^{\prime}\left(b_{+}\right)=S\left(b_{+}\right)$. So by 1 . and 2 . we deduce that $\Delta_{j+1}$ is 
obtained from $\Delta_{j}$ by attaching cones over the subcomplexes $T\left(b_{+}\right)$for $b_{+} \in R_{j+1}$. It will follow that $\pi_{1}\left(\Delta_{j}\right) \rightarrow \pi_{1}\left(\Delta_{j+1}\right)$ is an isomorphism for all $j \in \mathbb{N}_{0}$, by the Seifert-van Kampen theorem, provided that the $T\left(b_{+}\right)$are 1-connected.

To understand $T\left(b_{+}\right)$we use Lemma 4 . As $T\left(b_{+}\right)=\partial b_{+} * T^{\prime}\left(b_{+}\right) \cong \partial b_{+} * \Delta_{0}^{\prime}$, it suffices to show either that $\Delta_{0}^{\prime}$ is 1-connected, or that $\partial b_{+} \neq \varnothing$ and one of the pair $\partial b_{+}$and $\Delta_{0}^{\prime}$ is connected. We consider several cases.

- $\operatorname{rk} \Delta_{b_{+}}>$3: $\Delta^{\prime}$ satisfies the assumptions of the proposition, so $\Delta_{0}^{\prime}$ is 1connected by induction.

- $\mathrm{rk} \Delta_{b_{+}}=3$ : Again $\Delta_{0}^{\prime}$ is 1-connected, this time by hypothesis 2 .

- $\mathrm{rk} \Delta_{b_{+}}=2$ : Hypothesis 1 implies that $\Delta_{0}^{\prime}$ is connected. But rk $b_{+}+\operatorname{rk} \Delta_{b_{+}}=$ rk $\Delta>3$, so rk $b_{+} \geq 2$ and $\partial b_{+}$is nonempty.

- $\operatorname{rk} \Delta_{b_{+}}=1$ : This time $\mathrm{rk} b_{+}>2$, so $\partial b_{+}$is not only nonempty but also connected.

Finally, $\Delta_{+}$is 1-connected by the Solomon-Tits theorem, so

$$
\{1\}=\pi_{1}\left(\Delta_{+}\right)=\lim _{j \rightarrow \infty} \pi_{1}\left(\Delta_{j}\right)=\pi_{1}\left(\Delta_{0}\right) .
$$

Corollary 2. Suppose $\Delta$ is an irreducible, 3-spherical twin building, and every panel is contained in at least 6 chambers. Then $\Delta^{0}\left(C_{-}\right)$is 1-connected.

Proof. The assumptions on $\Delta$ imply that every rank-2 link is Moufang [3, Remark 5.212]. The fact that every panel is contained in at least 6 chambers then implies hypothesis 1. of Proposition 13 [2, Proposition 7].

Hypothesis 2. of Proposition 13 holds whenever the rank-3 link $\theta$ is irreducible, as explained in the proof of Theorem 2, once again using that every panel is contained in at least 6 chambers. If instead $\theta$ is reducible then $\theta^{0}(x)$ is a join of complexes of the same form but lower rank [2, Lemma 16], and therefore 1connected. 
Suppose $\left(G, B_{+}, B_{-}, N, S\right)$ is a twin BN-pair of irreducible 3-spherical type $(W, S)$. In the associated twin building $\Delta=\left(\Delta_{+}, \Delta_{-}\right)$, suppose every panel is contained in at least 6 chambers. Write $B_{ \pm}=G_{C_{ \pm}}$for opposite chambers $C_{ \pm} \in \Delta_{ \pm}$. As in Theorem 2, we have an action of $B_{-}$on $\Delta^{0}\left(C_{-}\right)$with $C_{+}$(together with its faces) as fundamental domain, and Corollary 2 paired with Proposition 12 yields

Corollary 3. Under the above assumptions on $\left(G, B_{+}, B_{-}, N, S\right)$ and $\Delta=\left(\Delta_{+}, \Delta_{-}\right), B_{-}$ is an amalgam of vertex stabilizers with respect to edge stabilizers of $C_{+}$.

We can phrase Corollary 3 in purely group-theoretic terms as follows.

Corollary 4. Let $\left(G, B_{+}, B_{-}, N, S\right)$ be a twin BN-pair of irreducible 3-spherical type and suppose that $\left[B_{ \pm}\{1, s\} B_{ \pm}: B_{ \pm}\right] \geq 6$ for all $s \in S$. Then $B_{-}$is the amalgam of its subgroups $B_{+}(S \backslash\{s\}) B_{+} \cap B_{-}(s \in S)$ along their intersections $B_{+}(S \backslash\{s, t\}) B_{+} \cap B_{-}(s \neq t \in S)$.

Proof. The index $\left[B_{ \pm}\{1, s\} B_{ \pm}: B_{ \pm}\right]$counts the number of chambers in the panel of cotype $s$ of the fundamental chamber $B_{ \pm}$in the building $\Delta\left(G, B_{ \pm}\right)$, hence in any panel of that cotype. We can therefore invoke Corollary 3. Its conclusion is the conclusion of this corollary, with $B_{+} \in \Delta\left(G, B_{+}\right)$playing the role of $C_{+}$.

\subsection{Finite presentability of $B_{ \pm}$}

We continue to work under the assumptions of Corollary 3. Let us also make some additional assumptions: that the action $G \stackrel{\phi}{\rightarrow}$ Aut $\Delta$ has finite kernel, and that $\Delta$ is locally finite in the sense that every panel is contained in finitely many chambers. We will show in this case that $B_{-}$is finitely presented.

let $\Sigma$ be the twin apartment containing the chambers $C_{ \pm}$. Set $H:=\operatorname{Fix}_{G} \Sigma=$ $B_{-} \cap B_{+}$. According to the rigidity theorem of Tits [3, Remark 5.208], any element of $H$ that fixes all chambers adjacent to $C_{+}$must lie in $\operatorname{ker} \phi$. As there are only finitely many such chambers, and $\operatorname{ker} \phi$ is finite, $H$ is finite as well.

We now prove that $B_{-}$is finitely presented by induction on $\operatorname{rk} \Delta$, beginning with the case $\mathrm{rk} \Delta=4$. Each stabilizer $\left(B_{-}\right)_{v}$ of a vertex of $C_{+}$acts on $\mathrm{lk}_{\Delta} v$, a 
finite building since $\Delta$ is 3-spherical and locally finite. The kernel of this action is contained in $H$, thus is finite, making $\left(B_{-}\right)_{v}$ finite as well. As an amalgam of finite groups, then, $B_{-}$is finitely presented.

Now comes the induction. If once more $v$ is a vertex of $C_{+}$, let $v_{-}$be its opposite in $\Sigma$, i.e. the vertex of $C_{-}$of matching type. We obtain a new twin BN-pair in $G^{\prime}:=G_{v} \cap G_{v_{-}}$of the form $\left(G^{\prime}, B_{+} \cap G^{\prime}, B_{-} \cap G^{\prime}\right)$ and associated twin building $\left(\Delta_{v}, \Delta_{v_{-}}\right)$; these satisfy the assumptions of Proposition 13 as well as the finiteness assumptions of this section, so $B_{-} \cap G^{\prime}$ is finitely presented by induction. But $B_{-} \cap$ $G^{\prime}=B_{-} \cap G_{v} \cap G_{v_{-}}=B_{-} \cap G_{v}=\left(B_{-}\right)_{v}$. Likewise, if $w$ is another vertex of $C_{+}$then $\left(B_{-}\right)_{v} \cap\left(B_{-}\right)_{w}=\left(B_{-}\right)_{\{v, w\}}=B_{-} \cap G^{\prime \prime}$ belongs to the BN-pair $\left(G^{\prime \prime}, B_{+} \cap G^{\prime \prime}, B_{-} \cap G^{\prime \prime}\right)$ in $G^{\prime \prime}:=G_{\{v, w\}} \cap G_{\mathrm{op}_{\Sigma}\{v, w\}}$ and is thus finitely presented by induction. We conclude that $B_{-}$is finitely presented, being an amalgam of finitely presented groups with respect to finitely presented subgroups.

In view of the manifest symmetry of + and - , the same conclusion holds for $B_{+}$.

Let us specialize to the case of Kac-Moody groups, in the sense of Tits [14].

Theorem 3. Let $\mathrm{G}$ be a Kac-Moody group functor of irreducible, 3-spherical type. Then the subgroups $B_{ \pm} \leq G\left(\mathbb{F}_{q}\right)$ are finitely presented if $5 \leq q<\infty$.

Remark. A similar result (finite presentation of parabolic subgroups when $7 \leq q<$ $\infty)$ was announced in [1, Theorem 2], with a sketch of a proof along the lines of this chapter. See also [8, Corollary 1.2].

Proof. It is well known that $G\left(\mathbb{F}_{q}\right)$ possesses an RGD system, and hence twin BNpair. We only need to verify the conditions in the preceding discussion. The root groups in $G\left(\mathbb{F}_{q}\right)$ are isomorphic to the additive group of $\mathbb{F}_{q}$, so in the associated twin building, each panel is contained in $q+1$ chambers, where $6 \leq q+1<\infty$. Moreover, the kernel of the action on the twin building is finite, being a subgroup of a finite torus $T\left(\mathbb{F}_{q}\right)$ [3, Proposition 8.82]. 


\section{Chapter 4}

\section{Some remarks on type $\mathrm{F}_{4}$}

The simple connectivity or sphericity of $\Delta^{0}(C)$ for buildings of exceptional types $\mathrm{F}_{4}, \mathrm{E}_{6}, \mathrm{E}_{7}$, and $\mathrm{E}_{8}$ remains open. In this chapter we report a coordinate description of the $\mathrm{F}_{4}$ geometry associated with a field. We manage to recognize opposition within this description, as well as understand certain links.

\subsection{The $\mathrm{E}_{6}$ and $\mathrm{F}_{4}$ geometries}

Here we summarize the construction of projective embeddings of the $E_{6}$ and $F_{4}$ geometries given in $[6, \S \S 5.2-5.3]$ and described more thoroughly in [5, ch. 18], to which named results in this section refer. Let $k$ be a field and $\mathbb{K}=M_{3}(k) \oplus M_{3}(k) \oplus$ $M_{3}(k)$. $\mathbb{K}$ carries the following structure:

- the cubic form (that is, homogeneous cubic polynomial function of the entries) $D: \mathbb{K} \rightarrow k$ given by $D\left(x_{1}, x_{2}, x_{3}\right)=\operatorname{det} x_{1}+\operatorname{det} x_{2}+\operatorname{det} x_{3}-\operatorname{tr} x_{1} x_{2} x_{3}$

- the symmetric trilinear form $(\cdot, \cdot, \cdot): \mathbb{K} \times \mathbb{K} \times \mathbb{K} \rightarrow k$ given by $(x, y, z)=$ $D(x+y+z)-D(x)-D(y)-D(z)+D(x+y)+D(x+z)+D(y+z)$

- the map $\#: \mathbb{K} \rightarrow \mathbb{K}$ given by $\left(x_{1}, x_{2}, x_{3}\right)^{\#}=\left(\operatorname{adj} x_{1}-x_{2} x_{3}, \operatorname{adj} x_{3}-x_{1} x_{2}, \operatorname{adj} x_{2}-\right.$ $\left.x_{3} x_{1}\right)$, where adj is the "classical adjoint" (transpose of the cofactor matrix). The entries of $\left(x_{1}, x_{2}, x_{3}\right)^{\#}$ are homogeneous quadratic polynomials in the entries of $\left(x_{1}, x_{2}, x_{3}\right)$. 
- the symmetric bilinear product $\times: \mathbb{K} \times \mathbb{K} \rightarrow \mathbb{K}$ given by $x \times y=(x+y)^{\sharp}-$ $x^{\sharp}-y^{\sharp}$

- the nondegenerate symmetric bilinear form $(\cdot, \cdot): \mathbb{K} \times \mathbb{K} \rightarrow k$ given by

$$
\left(\left(x_{1}, x_{2}, x_{3}\right),\left(y_{1}, y_{2}, y_{3}\right)\right)=\operatorname{tr}\left(x_{1} y_{1}+x_{2} y_{3}+x_{3} y_{2}\right)
$$

We record some identities relating these operations, taken from Proposition 18.1.8.

Proposition 14. The following identities hold for all $x, y, z, u \in \mathbb{K}$.

1. $(x \times y, z)=(x, y, z)$

2. $(x \times y) \times(x \times z)+x^{\sharp} \times(y \times z)=\left(x^{\sharp}, y\right) z+\left(x^{\sharp}, z\right) y+(x, y, z) x$

3. if $x^{\sharp}=0$ then $x \times(u \times(x \times z))=(x, u) x \times z$

It is not obvious from the definitions that $(\cdot, \cdot, \cdot)$ and $\times$ are multilinear; rather, these properties follow from $(1)$. Since $(\cdot, \cdot)$ is bilinear, $(1)$ implies that $(\cdot, \cdot, \cdot)$ is linear in its third argument, hence in all three arguments by the visible symmetry of $(\cdot, \cdot, \cdot)$. As for $\times$, we have

$$
\begin{aligned}
\left(\left(a x+x^{\prime}\right) \times y, z\right) & =\left(a x+x^{\prime}, y, z\right) \\
& =a(x, y, z)+\left(x^{\prime}, y, z\right) \\
& =a(x \times y, z)+\left(x^{\prime} \times y, z\right) \\
& =\left(a(x \times y)+\left(x^{\prime} \times y\right), z\right)
\end{aligned}
$$

for all $x, x^{\prime}, y, z \in \mathbb{K}$ and $a \in k$. Since $(\cdot, \cdot)$ is nondegenerate it follows that $\left(a x+x^{\prime}\right) \times$ $y=a(x \times y)+\left(x^{\prime} \times y\right)$. Linearity in the second argument follows by symmetry.

We write $\mathbf{P}(\mathbb{K})$ for the projective space over $\mathbb{K}$ and use angle brackets \langle\rangle to denote the span of a subset of $\mathbb{K}$ in $\mathbf{P}(\mathbb{K})$. We now define an incidence system having six types of elements, beginning with

$$
\text { - } P=\left\{\langle x\rangle \in \mathbf{P}(\mathbb{K}): x^{\sharp}=0\right\} \text { (points) }
$$


- $L=\{$ projective lines in $\mathbf{P}(\mathbb{K})$ consisting entirely of points in $P\}$ (lines)

The points $\langle x\rangle,\langle y\rangle \in P$ are collinear if and only if $(x+a y)^{\sharp}=x \times a y=a(x \times y)=0$ for all $a \in k$, which happens if and only if $x \times y=0$. We define the next three types of elements as singular subspaces of the space $(P, L)$.

- $V=\{$ singular subspaces of dimension 2$\}$ (planes)

- $M^{(4)}=\{$ maximal singular subspaces of dimension 4$\}$ (4-spaces)

- $M^{(5)}=\{$ maximal singular subspaces of dimension 5$\}$ (5-spaces)

Here "maximal" is with respect to containment among singular subspaces of $(P, L)$ and "dimension" refers to dimension as a projective subspace of $\mathbf{P}(\mathbb{K})$. According to Proposition 18.7.2(iv), every maximal singular subspace of $(P, L)$ is either 4- or 5-dimensional. An example of an element of $M^{(5)}$ is

$$
\left[\left(\begin{array}{lll}
0 & 0 & 0 \\
0 & 0 & 0 \\
0 & 0 & 0
\end{array}\right),\left(\begin{array}{lll}
0 & 0 & * \\
0 & 0 & * \\
0 & 0 & *
\end{array}\right),\left(\begin{array}{lll}
* & * & * \\
0 & 0 & 0 \\
0 & 0 & 0
\end{array}\right)\right] ;
$$

an example of an element of $M^{(4)}$ is

$$
\left[\left(\begin{array}{lll}
0 & 0 & 0 \\
0 & 0 & 0 \\
0 & 0 & 0
\end{array}\right),\left(\begin{array}{lll}
0 & 0 & * \\
0 & 0 & * \\
0 & 0 & *
\end{array}\right),\left(\begin{array}{lll}
* & 0 & 0 \\
* & 0 & 0 \\
0 & 0 & 0
\end{array}\right)\right] .
$$

Here and elsewhere this notation indicates the set of all points in $P$ which are spanned by vectors of the form depicted. Part of the assertion in the above examples is that every $x \neq 0$ of that form actually spans a point in $P$, i.e. has $x^{\sharp}=0$.

Finally, for $\langle x\rangle \in P$ we define $S_{x}=\{\langle x \times y\rangle \in P: y \in \mathbb{K}\}$ and

- $S=\left\{S_{x}:\langle x\rangle \in P\right\}$ (symps)

For any $\langle x\rangle,\langle y\rangle \in P$, either $x \times y=0$ or $\langle x \times y\rangle \in P$. This follows from the fact that $(x \times y)^{\sharp}=0$ whenever $x^{\sharp}=0$ and $y^{\sharp}=0$, which is for instance a consequence 
of Proposition 18.1.8(vi). In fact, more is true:

Proposition 15. Let $\langle x\rangle,\langle y\rangle \in P$.

1. (Lemma 18.7.1) If $\langle x\rangle$ and $\langle y\rangle$ are noncollinear then they belong to the unique symp $S_{x \times y}$.

2. (Lemma 18.7.2(vi)) $x \times y \neq 0$ if and only if $S_{x} \cap S_{y}$ is a single point, which is then $\langle x \times y\rangle$.

Two elements are declared incident if one contains the other, except in two cases. A 4-space and a 5-space are incident if their intersection is a 3-dimensional singular subspace (e.g. the 4-space and 5-space above), and a symp and a 5-space are incident if their intersection is a 4-dimensional singular subspace (which is necessarily nonmaximal).

The flag complex of the incidence system just defined is the building of type $\mathrm{E}_{6}$ over $k$ (Theorem 18.7.5).

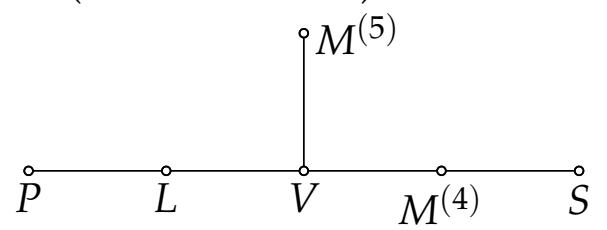

The map $\sigma: P \rightarrow S$ given by $\langle x\rangle \mapsto S_{x}$ is bijective and extends to a duality of the $\mathrm{E}_{6}$ geometry, which interchanges opposite types (Lemma 18.7.3). For $Y \in$ $P \cup L \cup V \cup M^{(4)} \cup S$ we define $\sigma(Y)=\cap_{\langle y\rangle \in Y} S_{y}$, and for $Y \in M^{(5)}$ we define $\sigma(Y)=\cup_{\pi \in V, \pi \subseteq Y} \sigma(\pi)$. For example, the 4-space mentioned above is dual to the line $\left\langle e_{2 ; 23}, e_{2 ; 33}\right\rangle$, and the 5 -space is self-dual.

An element of the $\mathrm{E}_{6}$ geometry that is incident with its dual is called absolute. Letting $P_{\sigma}, L_{\sigma}, V_{\sigma}$, and $M_{\sigma}^{(5)}$ denote the absolute points, lines, planes, and 5-spaces, we obtain another incidence system $\mathcal{M}:=P_{\sigma} \cup L_{\sigma} \cup V_{\sigma} \cup M_{\sigma}^{(5)}$, with two elements incident if one contains the other. It is a metasymplectic space; $\Delta:=$ Flag $\mathcal{M}$ is the building of type $\mathrm{F}_{4}$ over $k$ (Theorem 18.8.4).

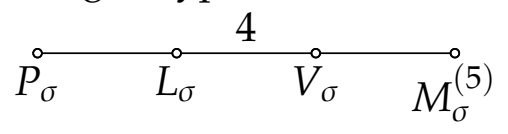


Following [16] we will refer to absolute 5-spaces as hyperlines when considering them as elements of the $\mathrm{F}_{4}$ geometry. Indeed they are not singular for this geometry but are isomorphic to polar spaces of rank 3 (Remark 18.8.5).

$\mathbb{K}$ has a standard basis $\left\{e_{i, j k}: 1 \leq i, j, k \leq 3\right\}$ of matrix units, each of which spans a point in $P$. The set $\left\{\left\langle e_{i, j k}\right\rangle: 1 \leq i, j, k \leq 3\right\}$ is called the standard frame of the $\mathrm{E}_{6}$ geometry and these points together with the elements they span [for symps, "span" should be interpreted in the sense of Proposition 15(1)] comprise the vertices of an apartment in the associated building. These points are all absolute except for $\left\langle e_{1 ; j j}\right\rangle$ $(1 \leq j \leq 3)$, and the absolute points constitute the standard frame of the $\mathrm{F}_{4}$ geometry and again define an apartment. In general a frame in the $\mathrm{E}_{6}$ or $\mathrm{F}_{4}$ geometry is the set of points in some apartment (its vertices of type "point").

For any $g \in G L(\mathbb{K})$ we may use the nondegeneracy of $(\cdot, \cdot)$ to define $g^{\sharp} \in G L(\mathbb{K})$ by the equation $\left(g x, g^{\sharp} y\right)=(x, y)$ for all $x, y \in \mathbb{K}$. Let $G=\{g \in G L(\mathbb{K}): D(g(x))=$ $D(x)$ for all $x \in \mathbb{K}\}$. Here are some facts about ${ }^{\sharp}$ and $G$.

Proposition 16 (Proposition 18.1.12). The map $g \mapsto g^{\sharp}$ determines an involutory automorphism of $G$ that satisfies the following equalities for all $g, h \in G$ and $x, y \in \mathbb{K}$.

1. $g^{\sharp \sharp}=g$

2. $(g h)^{\sharp}=g^{\sharp} h^{\sharp}$

3. $g^{\sharp}\left(x^{\sharp}\right)=(g x)^{\sharp}$

4. $g^{\sharp}(x \times y)=(g x) \times(g y)$

By (3), $x^{\sharp}=0$ if and only if $(g x)^{\sharp}=0$, and by (4), $x \times y=0$ if and only if $(g x) \times(g y)=0$. As a result, $G$ acts on the space $(P, L)$. If $\langle x \times y\rangle \in P$ then $\langle g(x \times y)\rangle \in P$, and by (1) and (4) we have

$$
g(x \times y)=g^{\sharp \sharp}(x \times y)=\left(g^{\sharp} x\right) \times\left(g^{\sharp} y\right),
$$

so $\langle g(x \times y)\rangle \in S_{g^{\sharp} x}$. Since $g$ is invertible this actually shows that $g S_{x}=S_{g^{\sharp} x}$. We conclude that $G$ acts on the $\mathrm{E}_{6}$ geometry, hence on its flag complex, and the latter 
action is strongly transitive (Exercise 18.9.24). Note that elements of $G$ need not preserve $(\cdot, \cdot), \times$, or $\sharp$; for instance, if there exists $1 \neq \omega \in k$ with $\omega^{3}=1$ then $x \mapsto \omega x$ preserves none of these.

Let $e=e_{1 ; 11}+e_{1 ; 22}+e_{1 ; 33}$ and consider the stabilizer $G_{e}$. This group acts on the $\mathrm{F}_{4}$ geometry in light of

Proposition 17 (Lemma 18.8.6). For $g \in G$ the following statements are equivalent.

1. $g e=e$

2. $g^{\sharp}=g$

3. $g \sigma=\sigma g$

Furthermore, $G_{e}$ acts strongly transitively on $\Delta$ (Exercise 18.9.26). Note that together with Proposition 16 and the equation $\left(g x, g^{\sharp} y\right)=(x, y)$, condition (2) above shows that $G_{e}$ does preserve $(\cdot, \cdot), \times$, and ${ }^{\sharp}$. The vector $e$ also plays another role:

Proposition 18 (cf. Lemma 18.8.1). The following are equivalent for a point $\langle x\rangle \in P$.

1. $\langle x\rangle \in P_{\sigma}$

2. $(x, x)=0$

3. $(x, e)=0$

4. $x \times e=-x$

Proof. To start with let us record an identity valid for arbitrary $x=\left(x_{1}, x_{2}, x_{3}\right) \in \mathbb{K}$. Using the definition of $\times$ we see that $x \times e=\left(\operatorname{adj}\left(x_{1}+I\right)-\operatorname{adj} x_{1}-I,-x_{2},-x_{3}\right)$, where $I \in M_{3}(k)$ is the identity matrix. After explicitly computing the first matrix component, this equation becomes

$$
\left.x \times e=\left[\begin{array}{ccc}
x_{1 ; 22}+x_{1 ; 33} & -x_{1 ; 12} & -x_{1 ; 13} \\
-x_{1 ; 21} & x_{1 ; 11}+x_{1 ; 33} & -x_{1 ; 23} \\
-x_{1 ; 31} & -x_{1 ; 32} & x_{1 ; 11}+x_{1 ; 22}
\end{array}\right),-x_{2,}-x_{3}\right],
$$


which may be reformulated as

$$
x=(x, e) e-x \times e
$$

since $(x, e)=\operatorname{tr} x_{1}$. We now proceed to the proof of the proposition.

$(1) \Rightarrow(2)$ : Write $x=x \times r$ for some $r \in \mathbb{K}$. Then using Proposition 14(1) and the symmetry of $(\cdot, \cdot, \cdot)$ we have $(x, x)=(x \times r, x)=(x, r, x)=(x, x, r)=(x \times x, r)=0$ since $x \times x=2 x^{\sharp}=0$.

(2) $\Rightarrow(3)$ : We compute

$$
\begin{aligned}
(x, e)^{2} & =(x, e)^{2}-(x \times x, e) \\
& =(x, e)^{2}-(x, x, e) \\
& =(x, e)^{2}-(x, e, x) \\
& =(x, e)(e, x)-(x \times e, x) \\
& =((x, e) e-x \times e, x) \\
& =(x, x) \\
& =0 .
\end{aligned}
$$$$
\text { by (*) }
$$

(3) $\Leftrightarrow(4)$ : Immediate from (*).

$(4) \Rightarrow(1):\langle x\rangle=\langle-x\rangle=\langle x \times e\rangle \in S_{x}$.

\subsection{Opposition}

Proposition 19 (Fact 3.2 of [16]). Given a point $x$ and a symp $\Sigma$, then either $x \in \Sigma$, or $x$ is opposite $\Sigma$, which is equivalent with "no point of $\Sigma$ is collinear with $x$ ", or there is a unique 5-space $V$ incident with both $x$ and $\Sigma$. In the latter case, the intersection of $V$ with $\Sigma$ is precisely the set of points of $\Sigma$ collinear with $x$, and we say $\Sigma$ neighbors $x$.

Opposition between points and symps has a simple algebraic description.

Proposition 20. The following are equivalent for points $\langle x\rangle,\langle u\rangle \in P$. 
1. $S_{x}$ is opposite $\langle u\rangle$.

2. $S_{u}$ is opposite $\langle x\rangle$.

3. $(x, u) \neq 0$.

Proof. Duality implies the equivalence of (1) and (2). Equivalence with (3) will follow from Proposition 14(3), which states

$$
x \times(u \times(x \times z))=(x, u) x \times z \text { for all } z \in \mathbb{K} .
$$

If $(x, u) \neq 0$ then for any $\langle x \times z\rangle \in S_{x}$ we have $x \times(u \times(x \times z)) \neq 0$, so $u \times(x \times z) \neq 0$ as well, meaning that $\langle u\rangle$ and $\langle x \times z\rangle$ are noncollinear, and $S_{x}$ is opposite $\langle u\rangle$ by Proposition 19. Conversely, if (1) and (2) hold then $x \times(u \times(x \times z)) \neq 0$ whenever $\langle x \times z\rangle \in S_{x}$ (Proposition 19 applied twice) and thus $(x, u) \neq 0$.

A model for the thin $\mathrm{F}_{4}$ geometry is the regular 4-polytope with Schläfli symbol $\{3,4,3\}$, also known as the 24-cell. More precisely, considering its 24 vertices as a set of points and declaring the pairs, triples, and 6-tuples of vertices of the edges, faces, and cells to be lines, planes, and hyperlines, we obtain an incidence system whose flag complex is the Coxeter complex of type $F_{4}[13, p .215]$. We will have in mind this structure (specifically its collinearity graph) when making arguments involving apartments of $\Delta$.

Proposition 21 ((M5) of [15]). Let $x$ and $y$ be two points in a metasymplectic space. Then one of the following situations occurs:

0. $x=y$.

1. There is a unique line incident with both $x$ and $y$. In this case we call $x$ and $y$ collinear and we denote the unique line by $x y$.

2. There is a unique hyperline incident with both $x$ and $y$. In this case there is no line incident with both $x$ and $y$, and we call $x$ and $y$ cohyperlinear. We denote the unique hyperline by $x \diamond y$. 
3. There is a unique point $z$ collinear with both $x$ and $y$. In this case we call $x$ and $y$ almost opposite and we denote $z$ by $x \bowtie y$.

4. There is no point collinear with both $x$ and $y$. In this case we call $x$ and $y$ opposite.

By convention, per section 2.1, we will refer to $x$ and $y$ as collinear in case (0) as well. Fortunately, the algebraic structure again furnishes simple descriptions of these cases.

Proposition 22. Let $\langle x\rangle$ and $\langle y\rangle$ be distinct absolute points. Then, in the $\mathrm{F}_{4}$ geometry, $\langle x\rangle$ and $\langle y\rangle$ are

1. collinear if and only if $\langle x\rangle \in S_{y}$, or equivalently $\langle y\rangle \in S_{x}$;

2. cohyperlinear if and only if $\langle x\rangle \notin S_{y}$ (equivalently, $\langle y\rangle \notin S_{x}$ ) and $x \times y=0$;

3. almost opposite if and only if $x \times y \neq 0$ and $(x, y)=0$, and in this case $\langle x \times y\rangle$ is the unique point collinear with both $\langle x\rangle$ and $\langle y\rangle$;

4. opposite if and only if $(x, y) \neq 0$.

Proof. Statement (1) is Lemma 18.8.2 in [5]. For the remaining statements, assume $\langle x\rangle$ and $\langle y\rangle$ are noncollinear and consider a frame containing them. Let $n$ be the number of points in the frame collinear with both $\langle x\rangle$ and $\langle y\rangle$; then $n=4$ if $\langle x\rangle$ and $\langle y\rangle$ are cohyperlinear, $n=1$ if almost opposite, and $n=0$ if opposite. As the criteria $\langle x\rangle \in S_{y},\langle x\rangle \notin S_{y}, x \times y=0, x \times y \neq 0,(x, y)=0$, and $(x, y) \neq 0$ appearing in (1)-(4) are all preserved by $G_{e}$, which acts strongly transitively, we may assume that $x=e_{2 ; 33}$ and the chosen frame is the standard frame. On one hand, the criteria can be readily checked in this case; in particular the symps have a simple shape, for instance

$$
S_{e_{2 ; 33}}=\left[\left(\begin{array}{lll}
0 & 0 & 0 \\
0 & 0 & 0 \\
* & * & *
\end{array}\right),\left(\begin{array}{lll}
0 & 0 & * \\
0 & 0 & * \\
0 & 0 & *
\end{array}\right),\left(\begin{array}{lll}
* & * & 0 \\
* & * & 0 \\
0 & 0 & 0
\end{array}\right)\right] .
$$

On the other hand, using (1) we can compute $n$ and thus verify (2)-(4). 
Since two elements $U, U^{\prime} \in \mathcal{M}$ are opposite if and only if they have the same type and every point of one has an opposite in the other, Proposition 22(4) has the following generalization.

Corollary 5. The elements $U$ and $U^{\prime}$ of $\mathcal{M}$ are opposite if and only if $(\cdot, \cdot)$ restricts to a perfect pairing $U \times U^{\prime} \rightarrow k$.

We will also need to understand the possible positions of lines, planes, and hyperlines relative to points.

Proposition 23 ((M7) of [15]). Let $x$ be a point and $h$ a hyperline of a metasymplectic space. Then one of the following situations occurs:

0. $x \in h$.

1. There is a unique line $l$ in $h$ such that $x$ is collinear with all points of l. Every point $y$ of $h$ which is collinear with all points of $l$ is cohyperlinear with $x$ and $x \diamond y$ contains 1. Every other point $z$ of $h$ (i.e., every point $z$ of $h$ collinear with a unique point $z^{\prime}$ of l) is almost opposite $x$ and $x \bowtie z=z^{\prime} \in l$.

2. There is a unique point $u$ of $h$ cohyperlinear with $x$. We have $h \cap(x \diamond u)=\{u\}$. All points $v$ of $h$ collinear with $u$ are almost opposite $x$ and $x \bowtie v \notin h$. All points $w$ of $h$ cohyperlinear with $u$ are opposite $x$.

\subsection{Viewing $\mathcal{M}^{>\langle q\rangle}$ as a classical polar space}

Let $\langle q\rangle \in P_{\sigma}$. From diagram considerations we know that $\mathcal{P}:=\mathcal{M}^{>\langle q\rangle}$ has the structure of a polar space of rank 3 . By this we mean that if we identify an element in $\mathcal{P}$ with the set of hyperlines that contain it, we obtain a space $\left(M_{\sigma}^{(5)} \cap \mathcal{P}, V_{\sigma} \cap \mathcal{P}\right)$ that is an abstract polar space of rank 3 as defined in section 2.1.

To make this concrete, fix some $\langle p\rangle \in P_{\sigma}$ opposite $\langle q\rangle$. According to Proposition 23, any hyperline $h \in \mathcal{P}$ contains a unique point $\left\langle u_{h}\right\rangle$ that is cohyperlinear with $\langle p\rangle$, and $h=\langle q\rangle \diamond\left\langle u_{h}\right\rangle$. In other words, there is a bijective correspondence $M_{\sigma}^{(5)} \cap \mathcal{P} \leftrightarrow W$, where $W$ is the set of absolute points cohyperlinear with both $\langle p\rangle$ 
and $\langle q\rangle$. For $\langle u\rangle,\left\langle u^{\prime}\right\rangle \in W$, we see from looking at an apartment (fig. 4-1) that $\langle q\rangle \diamond\langle u\rangle$ and $\langle q\rangle \diamond\left\langle u^{\prime}\right\rangle$ are opposite in Flag $\mathcal{P}$ exactly when $\langle u\rangle$ and $\left\langle u^{\prime}\right\rangle$ are opposite in $\Delta$, i.e. when $\left(u, u^{\prime}\right) \neq 0$ by Proposition 22. Since points in a polar space are either opposite or collinear, the collinearity relation on $\left(M_{\sigma}^{(5)} \cap \mathcal{P}, V_{\sigma} \cap \mathcal{P}\right)$ is therefore given by

$$
h \perp h^{\prime} \Leftrightarrow\left(u_{h}, u_{h^{\prime}}\right)=0 .
$$

Let us recall some notions from linear algebra. Given a $k$-vector space equipped with a reflexive bilinear form $f: V \times V \rightarrow k$, we may define for any subset $M \subseteq V$ its orthogonal complement $M^{\perp}:=\{x \in V: f(x, m)=0$ for all $m \in M\}$. A subspace $U \leq V$ is called totally isotropic if $U \subseteq U^{\perp}$.

Having in mind Propositions 18 and 22, we are led to define $V:=\{x \in \mathbb{K}$ : $x \times p=0, x \times q=0,(x, e)=0\}$, a linear subspace of $\mathbb{K}$. Then $W=\{\langle x\rangle: x \in$ $\left.V \backslash\{0\}, x^{\sharp}=0\right\}$. (Note: we don't need to specify $\langle x\rangle \notin S_{p}$ or $\langle x\rangle \notin S_{q}$ in defining $V$. These conditions hold automatically since $\langle p\rangle$ and $\langle q\rangle$ are opposite. A point $\langle x\rangle$ collinear with $\langle p\rangle$ is opposite or almost opposite $\langle q\rangle$ and hence has $x \times q \neq 0$, as can be seen by considering an apartment containing $\langle p\rangle$ and $\langle q\rangle$. Likewise $\langle x\rangle \in S_{q}$ implies $x \times p \neq 0$.) We claim that for all $x \in V, x^{\sharp}=0$ if and only if $(x, x)=0$, and hence $W$ is the set of isotropic lines in $V$ with respect to $(\cdot, \cdot)$.

Let us begin by assuming that $q=e_{2 ; 33}$ and $p=e_{3 ; 33}$, which satisfy $(p, q)=1$ and $p \times q=-e_{1 ; 33}$. So $V=V_{0}:=\left\{x \in \mathbb{K}: x \times e_{3 ; 33}=0, x \times e_{2 ; 33}=0,(x, e)=0\right\}$. After doing some computations using the definition of $\times$, one determines that $V_{0}$ consists of all vectors of the form

$$
x=\left[\left(\begin{array}{ccc}
x_{1 ; 11} & x_{1 ; 12} & 0 \\
x_{1 ; 21} & -x_{1 ; 11} & 0 \\
0 & 0 & 0
\end{array}\right),\left(\begin{array}{ccc}
0 & 0 & 0 \\
0 & 0 & 0 \\
x_{2 ; 31} & x_{2 ; 32} & 0
\end{array}\right),\left(\begin{array}{ccc}
0 & 0 & x_{3 ; 13} \\
0 & 0 & x_{3 ; 23} \\
0 & 0 & 0
\end{array}\right)\right],
$$

making $\operatorname{dim} V_{0}=7$. The restriction of $(\cdot, \cdot)$ to $V_{0}$ is given by

$(x, y)=2 x_{1 ; 11} y_{1 ; 11}+x_{1 ; 21} y_{1 ; 12}+x_{1 ; 12} y_{1 ; 21}+x_{3 ; 13} y_{2 ; 31}+x_{2 ; 31} y_{3 ; 13}+x_{3 ; 23} y_{2 ; 32}+x_{2 ; 32} y_{3 ; 23}$ 
which is visibly nondegenerate provided char $k \neq 2$, and we will assume this from now on. Because of the nondegeneracy, the Witt index of $(V,(\cdot, \cdot))$ is at most $\lfloor 7 / 2\rfloor=3\left[10\right.$, Theorem 6.11] and in fact is 3 since $\left\{e_{1 ; 21}, e_{3 ; 13}, e_{3 ; 23}\right\}$ spans a totally isotropic subspace.

One can check that

$$
x^{\sharp}=\frac{1}{2}(x, x) e_{3 ; 33} \times e_{2 ; 33} \text { for all } x \in V_{0}
$$

by a straightforward if tedious computation of both sides using the definitions of $\#$ and $(\cdot, \cdot)$, thus proving our claim in the special case $V=V_{0}$.

Now let $\langle p\rangle,\langle q\rangle \in P_{\sigma}$ be an arbitrary pair of opposite points. Since $G_{e}$ acts strongly transitively, there exists $g \in G_{e}$ with $g\left\langle e_{2 ; 33}\right\rangle=\langle q\rangle$ and $g\left\langle e_{3 ; 33}\right\rangle=\langle p\rangle$; by scaling $p$ and $q$ appropriately, we may assume $g e_{2 ; 33}=q$ and $g e_{3 ; 33}=p$. Recall that $G_{e}$ preserves $(\cdot, \cdot), \times$, and $\|^{\sharp}$. Then clearly $V=g V_{0}$, and the equation $x^{\sharp}=\frac{1}{2}(x, x) p \times q$ holds for all $x \in g V_{0}$, by applying $g$ to ( $\bullet$ ). The claim is now established, and we are ready to prove

Proposition 24. Assume char $k \neq 2$. Let $\langle p\rangle$ and $\langle q\rangle$ be opposite points of $\mathcal{M}$. Let $V=\{x \in \mathbb{K}: x \times p=0, x \times q=0,(x, e)=0\}$ and $X=\left\{0<U<V: U \subseteq U^{\perp}\right\}$, where " $\perp$ " is with respect to the trace form $(\cdot, \cdot)$. Then $V$ is 7-dimensional, $(\cdot, \cdot)$ is nondegenerate on $V$ of Witt index 3 , and $\Delta^{>\langle q\rangle} \cong$ Flag $X$.

Proof. The dimension, Witt index, and nondegeneracy claims were verified above for $\left(V_{0},(\cdot, \cdot)\right)$ and hold for its $G_{e}$-images as well, since $G_{e}$ acts by linear isomorphisms that preserve $(\cdot, \cdot)$. In this situation, $X$-or in our formalism, $(W,\{U \in X: \operatorname{dim} U=$ $2\}$ ) -is a polar space of $\operatorname{rank} 3\left[2\right.$, p. 81] in which $\langle u\rangle,\left\langle u^{\prime}\right\rangle \in W$ are collinear if and only if their span is totally isotropic, i.e. $\left(u, u^{\prime}\right)=0$. The isomorphism $\Delta^{>\langle q\rangle} \cong$ Flag $X$ will follow if we can show that this polar space is isomorphic to $\left(M_{\sigma}^{(5)} \cap \mathcal{P}, V_{\sigma} \cap \mathcal{P}\right)$.

We have seen that $\psi: h \mapsto\left\langle u_{h}\right\rangle$ provides a bijection $M_{\sigma}^{(5)} \cap \mathcal{P} \rightarrow W$. In view of (Co), the collinearity relations in the polar spaces coincide under $\psi$, and this is enough to conclude that $\psi$ is an isomorphism of spaces, since the lines of a nondegenerate polar space are determined by the collinearity relation [6, Theorem 


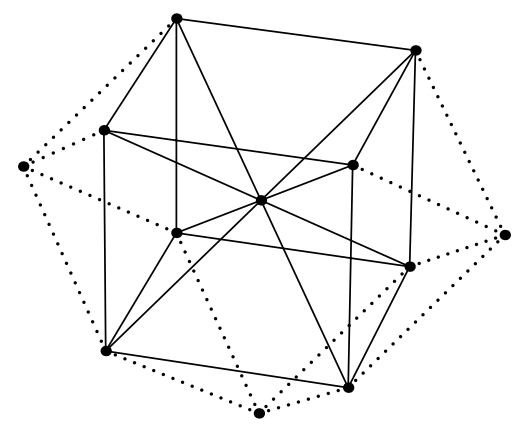

Figure 4-1: The solid lines form the collinearity graph of a point $\langle q\rangle$ (central dot) and its eight neighboring points in an apartment. Also shown, dotted, are three of the six hyperlines containing $\langle q\rangle$ in the apartment.

\section{1(iii)].}

Remark. The roles of $\langle p\rangle$ and $\langle q\rangle$ are of course symmetric in the above construction. This is a manifestation of the general fact that links of opposite simplices in a spherical building are isomorphic.

Let $\delta=$ Flag $X$. Subspaces $U$ and $W$ of $V$ are called transversal, written $U \pitchfork W$, if $U \cap W=0$ or $U+W=V$.

Proposition 25 (Lemma 29 of [2]). $U, E \in X$ are opposite in $\delta$ if and only if $V=U \oplus E^{\perp}$. $U \in \delta^{0}(E)$ holds if and only if $U \pitchfork E^{\perp}$.

\subsection{More results}

Proposition 26. Suppose the points $\langle p\rangle,\langle q\rangle,\langle\alpha\rangle \in P_{\sigma}$ are pairwise opposite. Then $\langle q \times$ $(p \times \alpha)\rangle \in P$, and the following are equivalent.

1. $\langle q\rangle \in S_{p \times \alpha}$

2. $\langle q\rangle=\langle q \times(p \times \alpha)\rangle$

3. $\langle q \times \alpha\rangle=\langle q \times p\rangle$

Proof. To prove the first claim it suffices to show that $q \times(p \times \alpha) \neq 0$. By Proposition 14(3), $p \times(q \times(p \times \alpha))=(p, q) p \times \alpha$. As $\langle p\rangle$ is opposite both $\langle q\rangle$ and $\langle\alpha\rangle$, 
$(p, q) \neq 0$ and $p \times \alpha \neq 0$ by Proposition 22, so $p \times(q \times(p \times \alpha)) \neq 0$ and thus $q \times(p \times \alpha) \neq 0$ as well. Now we prove the claimed equivalences.

$(1) \Rightarrow(2)$ : Since $\langle q\rangle$ is absolute we have $\langle q\rangle \in S_{q} \cap S_{p \times \alpha}=\{\langle q \times(p \times \alpha)\rangle\}$ according to Proposition 15(2).

(2) $\Rightarrow$ (3): By Proposition 14(3), $\langle q \times \alpha\rangle=\langle\alpha \times(q \times(p \times \alpha))\rangle=\langle(\alpha, q) p \times \alpha\rangle=$ $\langle p \times \alpha\rangle$. A similar calculation shows that $\langle q \times p\rangle=\langle p \times \alpha\rangle$.

(3) $\Rightarrow$ (1): Evidently $\langle q \times \alpha\rangle \in S_{p}$, whence $\langle p\rangle \in S_{q \times \alpha}$ by duality. We can then apply the preceding arguments, with the roles of $p$ and $q$ swapped, to obtain (1).

Under the assumption $x^{\sharp}=0,(1)$ and (2) of Proposition 14 have the following consequence.

Proposition 27. Let $z \in \mathbb{K}$. Then for any symp $S_{x}$ and any point $\langle x \times y\rangle \in S_{x},(x \times y, z)=$ 0 if and only if $(x \times y) \times(x \times z)=0$. 


\section{Bibliography}

[1] P. Abramenko. Finiteness properties of groups acting on twin buildings. In Groups: topological, combinatorial and arithmetic aspects, volume 311 of London Math. Soc. Lecture Note Ser., pages 21-26. Cambridge Univ. Press, Cambridge, 2004.

[2] Peter Abramenko. Twin buildings and applications to S-arithmetic groups, volume 1641 of Lecture Notes in Mathematics. Springer-Verlag, Berlin, 1996.

[3] Peter Abramenko and Kenneth S. Brown. Buildings, volume 248 of Graduate Texts in Mathematics. Springer, New York, 2008. Theory and applications.

[4] Garrett Birkhoff. Lattice Theory. American Mathematical Society, New York, 1940.

[5] Francis Buekenhout and Arjeh M. Cohen. Diagram Geometry.

[6] Arjeh M. Cohen. Point-line spaces related to buildings. In Francis Buekenhout, editor, Handbook of incidence geometry, pages 647-737. North-Holland, Amsterdam, 1995. Buildings and foundations.

[7] B De Bruyn and H Van Maldeghem. Non-embeddable polar spaces. Münster J. Math, 2013.

[8] Alice Devillers and Bernhard Mühlherr. On the simple connectedness of certain subsets of buildings. Forum Math., 19(6):955-970, 2007.

[9] M Horn, R Nessler, and H Van Maldeghem. Simple connectivity in polar spaces. 2014. submitted.

[10] Nathan Jacobson. Basic algebra. I. W. H. Freeman and Company, New York, second edition, 1985.

[11] Bernhard Mühlherr and Mark Ronan. Local to global structure in twin buildings. Invent. Math., 122(1):71-81, 1995.

[12] Christophe Soulé. Groupes opérant sur un complexe simplicial avec domaine fondamental. C. R. Acad. Sci. Paris Sér. A-B, 276:A607-A609, 1973.

[13] Jacques Tits. Buildings of spherical type and finite BN-pairs. Lecture Notes in Mathematics, Vol. 386. Springer-Verlag, Berlin, 1974. 
[14] Jacques Tits. Uniqueness and presentation of Kac-Moody groups over fields. J. Algebra, 105(2):542-573, 1987.

[15] Hendrik Van Maldeghem. Generalized polygons, volume 93 of Monographs in Mathematics. Birkhäuser Verlag, Basel, 1998.

[16] Hendrik Van Maldeghem. Symplectic polarities of buildings of type e 6 . Designs, Codes and Cryptography, 65:115-125, 2012. 